\title{
1 Scx-positive tendon cells are required for correct muscle patterning
}

3 Yudai Ono ${ }^{1}$, Tempei Sato ${ }^{2,3}$, Chisa Shukunami ${ }^{4}$ Hiroshi Asahara $^{3,5}$, Masafumi Inui ${ }^{1,2,6^{*}}$

5 1. Laboratory of Animal Regeneration Systemology, Department of Life Science, School

6 of Agriculture, Meiji University, Kanagawa, 214-8571, Japan

7 2. Department of Systems BioMedicine, National Research Institute for Child Health and

8 Development, Tokyo 157-8535, Japan

9 3. Department of Systems BioMedicine, Graduate School of Medical and Dental

10 Sciences, Tokyo Medical and Dental University, Tokyo 113-8510, Japan

11 4. Department of Molecular Biology and Biochemistry, Graduate School of Biomedical

12 and Health Sciences, Hiroshima University, Hiroshima, 734-8553, Japan.

13 5. Department of Molecular Medicine, The Scripps Research Institute, La Jolla, CA

1492037, USA

15 6. Meiji University International Institute for Bio-Resource Research, Kanagawa,

16 214-8571, Japan

17

18

$19 *$ Correspondence: inui_m@meiji.ac.jp 


\section{Summary}

23 The elaborate movement of the vertebrate body is supported by the precise connection of

24 muscle, tendon and bone. Each of the $>600$ distinct skeletal muscles in the human body

25 has unique attachment sites; however, the mechanism through which muscles are

26 reproducibly attached to designated partner tendons during embryonic development is

27 incompletely understood. We herein show that Screlaxis-positive tendon cells have an

28 essential role in correct muscle attachment in mouse embryos. Specific ablation of

29 Screlaxis-positive cells resulted in dislocation of muscle attachment sites and abnormal

30 muscle bundle morphology. Step-by-step observation of myogenic cell lineage revealed

31 that post-fusion myofibers, but not migrating myoblasts, require tendon cells for their

32 morphology. Furthermore, muscles could change their attachment site, even after the

33 formation of the insertion. Our study demonstrated an essential role of tendon cells in the

34 reproducibility and plasticity of skeletal muscle patterning, in turn revealing a novel

35 tissue-tissue interaction in musculoskeletal morphogenesis. 
37 Keywords: skeletal muscle, tendon, insertion, MTJ, limb, deltoid, gluteus, pectoralis,

38 Scx,

39

40 Graphical abstract

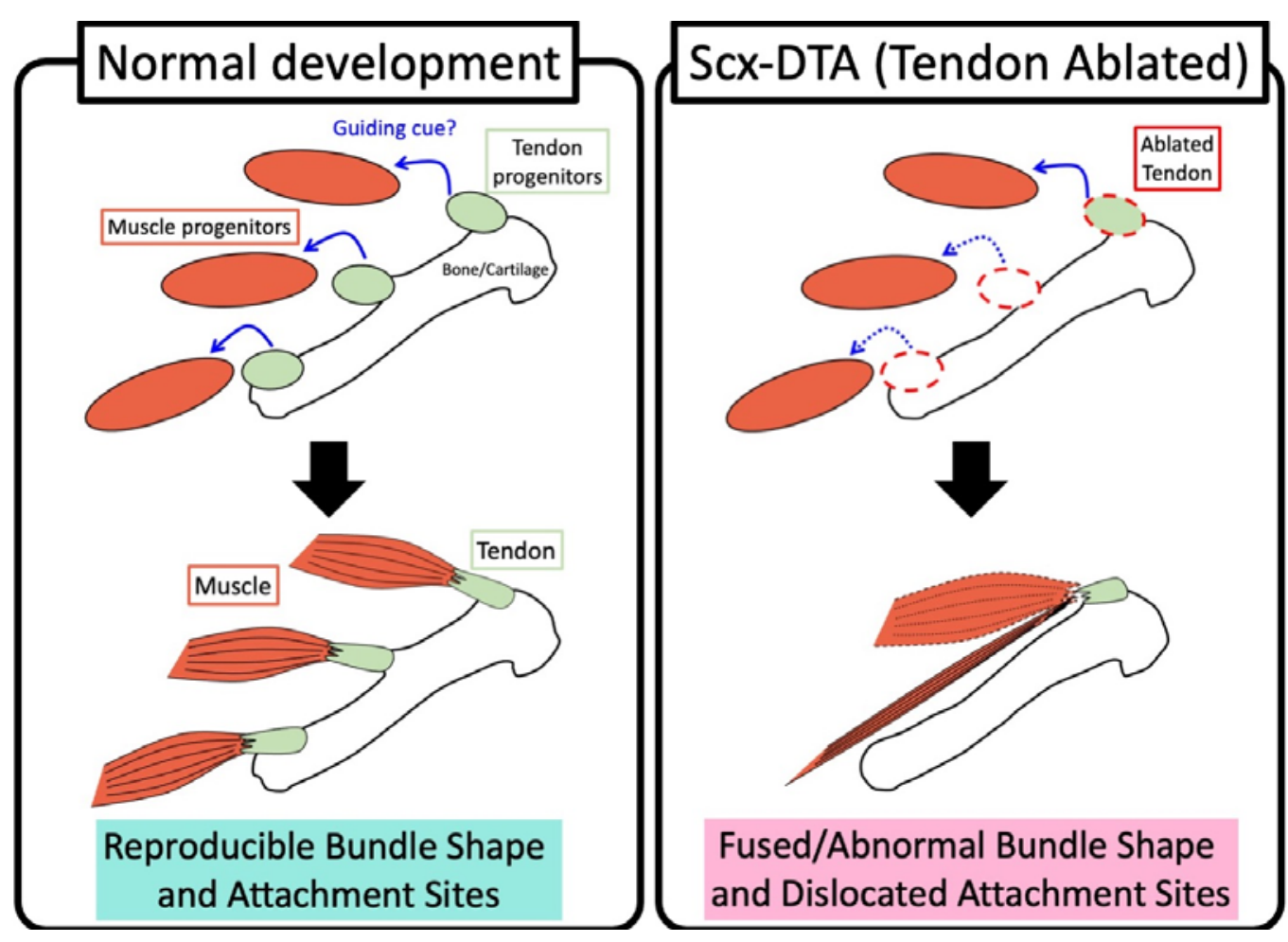




\section{Introduction}

44 The musculoskeletal system is a complex multi-tissue system that consists of muscle,

45 tendon and bone, as well as associating connective tissues. Elaborate body movements of

46 vertebrate are supported by the precise shape, position and functional connections of

47 these components. While the differentiation process of each component has been well

48 studied (Asahara, Inui, \& Lotz, 2017; Buckingham \& Rigby, 2014; Kozhemyakina,

49 Lassar, \& Zelzer, 2015), their tissue integration process remain largely unexplored.

50 Hundreds of skeletal muscles exist in the mammalian body; however, most are derived

51 from the somites. Myogenic progenitor cells migrate long distances to destinations such

52 as the limbs, form a precise bundle shape, and attach with appropriate tendons and bones

53 during embryonic development (Buckingham et al., 2003; Comai \& Tajbakhsh, 2014). It

54 is well known that the migration of myogenic progenitor cells from somite to limb bud is

55 guided by secreted cues such as HGF/SF or SDF-1 (Dietrich et al., 1999; Griffin, Apponi,

56 Long, \& Pavlath, 2010). However, mechanisms that regulate tissue integration between

57 post-migration muscle cells and tendons and bones are not fully understood (Kardon,

58 2011; Schweitzer, Zelzer, \& Volk, 2010). Considering the distinct cellular origin (i.e., 
59 muscles from the somites and tendons from the lateral plate) and large number of

60 integrations to be formed in limited time and space, it is reasonable to assume that the

61 local tissue-tissue interactions take place between myogenic progenitor cells and the

62 surrounding cell/tissue. Indeed, several factors, including transcription factors, ECM,

63 muscle connective tissue cells (MCTs) are reported to regulate the patterning of

64 post-migrating myogenic cells in vertebrate limbs (Besse et al., 2020; Hasson et al., 2010;

65 Helmbacher \& Stricker, 2020; Kardon, Harfe, \& Tabin, 2003; Kutchuk et al., 2015;

66 Rodriguez-Guzman, Montero, Santesteban, Gañan, Macias, \& Hurle, 2007b; Swinehart,

67 Schlientz, Quintanilla, Mortlock, \& Wellik, 2013). However, whether tendon cells, the

68 intrinsic partner of skeletal muscles, have any role in instructing the muscle shape and

69 patterning in mammals is unclear. To examine the role of tendon cells in the regulation of

70 muscle patterning, we took a simple approach of linage-specific cell ablation and reduced

71 the tendon cells in embryos. As a result, we found that muscle attachment patterns are

72 significantly altered in the embryo with reduced tendon cells. Our results indicate that

73 Scx-positive tendon cells have an important instructive role in spatially precise muscle

74 attachment patterns, and are in turn essential for reproducible and robust musculoskeletal 
bioRxiv preprint doi: https://doi.org/10.1101/2021.01.03.424463; this version posted January 4,2021 . The copyright holder for this preprint (which was not certified by peer review) is the author/funder, who has granted bioRxiv a license to display the preprint in perpetuity. It is made available under aCC-BY-NC-ND 4.0 International license.

75 morphogenesis.

76 


\section{Results and Discussion}

\section{ScxCre mediated tendon ablation}

80 (Sugimoto, Takimoto, Hiraki, \& Shukunami, 2013b) with a Rosa26-LSL-DTA mouse

81 (Voehringer, Liang, \& Locksley, 2008) (Fig. 1A). As a result, we observed

82 TUNEL-positive cells in tendon tissue of the tail and limb of Cre+/DTA+ embryo at

83 E15.5, but not in control embryos (Fig. 1C, E arrowheads, Fig. S1 A-D). H\&E staining of

84 consecutive sections showed tissue ablation in the tail tendon tissue of Cre+/DTA+

85 embryo (Fig. 1B, D arrowheads). Cre and DTA dependent TUNEL-positive cells were

86 observed as early as E12.5 in the forelimb, shoulder, intervertebral mesenchyme, or tail

87 (Fig. S1 E-P). These results suggest that we could successfully induce cell death

88 specifically in the tendon cells, from their early stage of differentiation. Hereafter, in this

89 manuscript, we designate the mice with ScxCre-L Tg and Rosa26-LSL-DTA alleles as

90 "Scx-DTA" mice.

91 Next, we examined the tendon tissue of Scx-DTA embryo with the Mkx-Venus

92 knock-in allele (Ito et al., 2010). As shown in Fig. 1F and J, the long tendons in the 
zeugopod were reduced in E17.5 Scx-DTA embryo. The limb sections showed that the

94 flexor digitorium profundus (FDP) tendon (Fig. 1G, K) and flexor digitorium sublimis

95 (FDS) tendon (Fig. 1H, L) in the autopod, extensor carpi radialis tendons, extensor

96 digitorium communis (EDC) tendons, or the palmaris longus tendon (Fig. 1I, M) in the

97 zeugopod were greatly reduced in Scx-DTA embryos. The reduction of tendon tissue in

98 other parts of the body, such as the Achilles tendon (Fig. 1N, R) or tail tendon (Fig. 1O, S)

99 was also apparent. Furthermore, ligamentous tissue, such as the cruciate and patella

100 ligaments were diminished (Fig. 1P, T) and the outer annulus fibrosus of the

101 intervertebral disc was also reduced (Fig. 1Q, U). In sum, these results illustrated that our

102 approach reduced the tendon and ligament tissues from the developing embryo, albeit not

103 completely. The reductions of tendon tissue have been reported in mice with the knockout

104 of tendon transcription factors (TFs), such as Scleraxis (Scx), Mohawk (Mkx) or Egr1

105 (Guerquin et al., 2013; Ito et al., 2010; Murchison et al., 2007). The degree of

106 tendon/ligament reduction in Scx-DTA mice was as severe as or even more severe than

107 those observed in these TF mutants. Namely, the cell death was observed as early as

108 E12.5 in Scx-DTA embryo, which is earlier than the reported tendon reduction in TF 
mutants. In addition, while the tendon reduction was seen mainly in the long tendons in

110 TF mutants, most of tendon and ligament tissues were reduced in Scx-DTA embryos. The

111 Incomplete loss of tendon tissue was probably due to the penetrance of Cre

112 activity(Comai, Sambasivan, Gopalakrishnan, \& Tajbakhsh, 2014), or continuous

113 recruitment of $S c x$-positive tendon cells from limb mesenchyme population (Huang et al.,

114 2019; Shwartz, Viukov, Krief, \& Zelzer, 2016). As tendon cell death occurs in a period

115 that overlaps the individuation of tendon from anlage (Huang et al., 2015), this could also

116 have an impact on tendon patterning. Indeed, the numbers of FDS or EDC tendons were

117 reduced and outline of each of the tendons were indistinct in the Scx-DTA embryo (Fig.

$1181 \mathrm{H}, \mathrm{I}, \mathrm{L}, \mathrm{M})$

119 We found that Scx-DTA pups died soon after birth (Fig. 1V, W). Scx-DTA

120 embryo showed a defect in diaphragm (described below), which could cause an

121 insufficient respiratory function in newborn Scx-DTA pups. In addition, as Scx is

122 expressed also in non-tendon/ligament tissues such as the patella, rib cage, and bone

123 ridges (Blitz, Sharir, Akiyama, \& Zelzer, 2013; Sugimoto, Takimoto, Akiyama, Kist,

124 Scherer, et al., 2013a), these Scx-positive skeletal elements are also reduced or lost in 
125 Scx-DTA embryos (Fig. 1X, Fig. S2). The loss of rib cage could also cause respiratory

126 problems. Thus, in the present study, we only focused on embryonic development. The

127 effects of skeletal abnormality are also discussed (see below).

129 Muscle patterning was altered in tendon-ablated embryos

130 Next, we examined whether the patterning (i.e., shape or attachment) of

131 skeletal muscles was altered in Scx-DTA embryos. First, we applied whole-mount

132 immunohistochemistry with a myosin heavy chain (MHC) antibody for the analysis of

133 the forelimb of P0 pups. In control limbs, the muscles were located between their regular

134 attachment sites (origin and insertion); for example, the deltoid muscle originates from

135 spine of the scapula and inserts into deltoid tuberosity (Fig. 2B, C blue arrowhead). In

136 contrast, muscles in Scx-DTA mice showed changes in their attachment sites; for example,

137 the insertion site of the deltoid muscle changed to the shoulder joint (Fig. 2F, G red

138 arrowhead). The shapes/attachments of muscles in the zeugopod also changed, for

139 example the extensor carpi ulnaris (ECU) and extensor digiti quarti/quinti (EDQ)

140 muscles were clearly separated in the control limb (Fig. 2D arrowheads), but they were 
141 fused into a single muscle bundle in the $S c x-D T A$ limb (Fig. 2H arrowhead). The extensor

142 pollicis muscles, which are normally covered by superficial muscles, became visible (Fig.

$1432 \mathrm{D}, \mathrm{H}$ arrows). Of note, despite their morphological change, most of the muscles in the

144 zeugopod attached with tendons at their distal end.

145 In sections of E18.5 forelimbs, muscles in the metacarpal position were grossly normal

146 (Fig. 2I, L, O). However, FDS muscles were found in the wrist position of the Scx-DTA

147 limb, where muscle was not observed in control embryos (Fig. 2J, M, O). Conversely,

148 FDS muscles were missing from their normal position in the zeugopod of Scx-DTA mice

149 (Fig. 2K, N, O arrowheads). Interestingly, similar distal dislocation of FDS muscle has

150 been reported in $S c x$-knockout mice (Huang et al., 2013), indicating that this change in

151 muscle position is due to the reduction of FDS and FDP tendons. In addition, the

152 arrangement of the extensor carpi (longus/Brevis) and FDP muscles was changed (Fig.

$1532 \mathrm{~K}, \mathrm{~N}$ arrows). On the other hand, muscles in the hindlimb generally showed a normal

154 attachment pattern (Fig. S3).

Altered attachment sites were also observed in other muscles, especially in

156 muscles connecting to the body trunk and limbs. For example, the pectoral muscles 
157 originate from the sternum and insert into the deltoid tuberosity in control embryos (Fig.

158 2P, P' arrowhead); however, the insertion sites of these muscles were distally dislocated

159 toward the elbow joint in Scx-DTA mice (Fig. 2R, R' arrowhead). In a severe case, the

160 pectoral minor muscle separated into two bundles (Fig. 2R, R' arrows). Similarly, in

161 Scx-DTA mice, the insertion site of the gluteus maximus muscle, which normally

162 originates from the ilium and inserts into the gluteus tuberosity (Fig. 2Q, Q' arrowheads)

163 changed distally, toward the knee joint (Fig. 2S, S'). The organization of muscles in the

164 trunk, such as the rectus abdominis, was also changed in Scx-DTA mice (Fig. 2T, V). In

165 the diaphragm, the width of the central tendon was greatly reduced (Fig. 2U, W arrows)

166 and the orientation of associated muscle fibers was changed (Fig. 2U, W arrowheads). In

167 sum, the organization of skeletal muscles of many body parts was changed in Scx-DTA

168 mice, suggesting that tendon cells are necessary for skeletal muscle to form a precise

169 shape and attachment in the correct position. The role of tendon tissue in muscle

170 patterning has been shown indirectly in other vertebrate species; for example, the surgical

171 removal of the tendon primordia resulted in ectopic extension of the muscle into the knee

172 joint in chicken embryos (Kardon, 1998). Scx-knockout zebrafish embryos showed 
173 abnormal muscle patterning (Kague et al., 2019). In mammals, muscle-tendon interaction

174 has been studied in the opposite direction and the requirement of muscle cells for tendon

175 formation has been reported (Brent, Braun, \& Tabin, 2005; Pryce et al., 2009). Our results

176 represent the first evidence that directly points to the importance of tendon cells in muscle

177 patterning in mammalian embryos. Muscle patterning in limbs also depends on

178 LPM-derived mesenchyme cells or muscle connective tissue (MCT) cells that are closely

179 associated with the myogenic lineage (Hasson et al., 2010; Kardon et al., 2003;

180 Vallecillo-García et al., 2017). As such, we considered whether MCT is affected in

181 Scx-DTA mice. As shown in Fig. S4, the expression levels of MCT marker genes were

182 unaltered in Scx-DTA mice, while tendon marker genes were clearly reduced. Also, Cre

183 expression in ScxCre- $L \mathrm{Tg}$ is restricted in tendon cells and not detected in MCTs

184 (Sugimoto, Takimoto, Hiraki, \& Shukunami, 2013b). We concluded that the phenotype

185 seen in Scx-DTA mice is primarily due to the loss of tendon cells.

187 Myofibers but not myoblasts require tendon cells for patterning

188 Next, to elucidate the stage at which tendon-muscle interaction takes place, we 
monitored the location and shape of the myogenic cell lineage in a step-by-step manner,

190 along with their migration and differentiation. First, we found that pax3-positive

191 myogenic progenitor cells were induced in the somite and migrated normally into the

192 limb buds at E11.5 (Fig.3A, D arrowheads). Myogenin-positive myoblast positions were

193 grossly normal at E12.5 or E13.5 (Fig.3B, C, E, F), suggesting that myoblast segregation

194 occurred in similar manner in control and Scx-DTA mice. Furthermore, myh3-positive

195 myofibers were observed in same position in Scx-DTA and control embryos at E12.5 (Fig.

$1963 \mathrm{G}, \mathrm{J})$, indicating that myofiber differentiation is not dependent on tendon cells. Of note,

197 we found that the myh3 signal became significantly high at the distal/proximal ends of

198 myofibers from E13.5 (Fig. 3H, I arrowheads). By comparing the expression of myh3 and

199 a mature tendon marker, tenomodulin (Tnmd) (Shukunami, Oshima, \& Hiraki, 2001;

200 Shukunami et al., 2018), we noted that this high myh3 expression marks the boundary of

201 muscle and tendon (Fig. S3). We therefore interpreted the myh3 high region as surrogate

202 of the muscle-tendon boundary. The shape of myh3-positive muscle bundles clearly

203 differed between Scx-DTA and control embryos at E13.5 (Fig.3H, K). While the EDC

204 muscle in the control embryo formed a sharp boundary at its distal tip (Fig. 3H), the distal 
205 tip of EDC in Scx-DTA embryo remained loose shape without the clear boundary

206 formation (Fig. 3K). Moreover, while the ECU and EDQ muscle bundles in control

207 embryos were clearly separated and formed two distal boundaries (Fig. 3H blue

208 arrowheads), those muscles did not separate or form normal distal boundaries in Scx-DTA

209 embryos (Fig. 3K red arrowheads). The muscle patterning defects became more apparent

210 in E14.5, where EDC/EDQ/ECU muscles could not be distinguished (Fig. 3I, L).

211 Immunohistochemistry with MHC antibodies confirmed that the shapes of MHC-positive

212 muscle bundles became loose in Scx-DTA embryos (Fig. 3M-P) and the insertion sites of

213 several muscles, including the triceps (Fig. 3 M, O) and deltoid muscles (Fig. 3N, P),

214 were dislocated. These results indicate that tendon cells are dispensable for the migration,

215 initial segregation, or differentiation of myofibers, but are required for the formation of

216 proper muscle bundle morphologies and the precise location of the attachment site.

217 Previous studies showed that tendon anlages are induced in muscles-less limbs normally;

218 however the maturation of tendons requires muscles (Brent et al., 2005; Huang et al.,

219 2015; Pryce et al., 2009). Our results together with those studies, indicate that muscle and

220 tendon anlage are induced independently, but their connection and maturation is mutually 
221 dependent. The patterning of limb muscle is regulated by surrounding tissues at various

222 time point (Helmbacher \& Stricker, 2020). While the loss of the Tbx5 in Prxl-positive

223 mesenchyme altered the patterning of tendon and muscle from E12.5 (Hasson et al.,

224 2010), Osr2Cre dependent MCT ablation affected the patterning of MyoD-positive

225 muscle clusters at E13.0 (Besse et al., 2020). Our results implied that the role of tendon

226 cells, which is to finalize the muscle bundle position and attachment, is played later in

227 comparison to other connective tissues. It is likely that multiple inputs from surrounding

228 cells regulate limb muscle patterning sequentially and in an overlapping manner.

\section{Skeletal malformation or cell death are not the cause of muscle patterning defects}

As $S c x$ is also expressed in some skeletal elements, such as the rib and patella

232 (Sugimoto, Takimoto, Hiraki, \& Shukunami, 2013b), Scx-DTA mice showed several

233 skeletal malformations, including loss of the rib cage or deltoid tuberosity (Fig. 1X, Fig.

234 S2). To exclude the possibility that these skeletal malformation caused the muscle

235 patterning defects, we examined the attachment pattern in embryos in which skeletal

236 defects similar to those of Scx-DTA mice were reported (Braun, Rudnicki, Arnold, \& 
237 Jaenisch, 1992; Kist et al., 2002). Sox9 heterozygous (Sox $9^{f /-}$ ) embryos which lack the

238 deltoid tuberosity were generated by mating Sox $9^{\text {flox/flox }}$ and Meox2-Cre mice (Fig. S6B,

239 D). Heterozygous $M y f 5^{C r e /+}$ knock-in mice were intercrossed to generate homozygous

240 Myf5 $5^{\text {Cre/Cre }}$ embryos, which resulted in rib cage malformation due to the loss of the Myf5

241 protein (Fig. S5F, H). In both cases the insertion sites of the pectoralis major muscles

242 were not altered, despite the skeletal defects (Fig. S6 A, C, E, G). These results imply that

243 the loss of skeletal elements is not the major cause of the muscle patterning defect seen in

$244 S c x$-DTA mice. A previous study on the role of transcription factor $L m x 1 b$ also showed

245 that the change of skeletal elements alone does not cause the muscle patterning change.

246 Deletion of LmxIb in a Prxl-positive lineage altered the dorso-ventral polarity of the

247 whole limb, including the bone, tendon and muscle; however the loss of Lmxlb in the

248 Sox9-positive lineage resulted in the change of bone, but not tendon or muscle polarity

249 (Li, Qiu, Watson, Schweitzer, \& Johnson, 2010).

250 Programmed cell death is one of the important driving forces of morphogenesis,

251 such as digit formation or muscle belly segregation (Guha, Gomes, Kobayashi, Pestell, \&

252 Kessler, 2002; Rodriguez-Guzman, Montero, Santesteban, Gañan, Macias, \& Hurle, 
253 2007a). As such, the cell death induced in Scx-DTA mice could directly or indirectly

254 affect the muscle attachment pattern. To explore this possibility, we examined the muscle

255 patterning of the embryo where cell death is induced in muscle tissue by the Myf5-Cre

256 dependent expression of DTA $\left(M y f 5^{C r e /+}:\right.$ Rosa26-LSL-DTA, hereafter "Myf5-DTA"). In

257 Myf5-DTA embryos, despite a severe reduction of muscle mass, the remaining pectoralis

258 major muscle was correctly inserted into the deltoid tuberosity (Fig. S6I, J). This result

259 indicated that excess cell death in the tissue does not necessarily cause muscle patterning

260 defects.

261

262 Dynamic change in muscle patterning after myofiber formation

263 The results shown above implied that the skeletal muscles define their

264 attachment sites after myofiber formation (e.g., Fig. 3 G-L). We then asked if the position

265 of muscle could be altered after the formation of insertion, which may provide skeletal

266 muscle patterning further plasticity and robustness. To examine this point, we observed

267 the position and insertion of gluteus maximus muscle at two time points E14.5 and E17.5.

268 As shown in Fig. 4A and D, the distal tips of the gluteus maximus are located at the 
middle of the femur (i.e., the gluteus tuberosity) at E14.5 in both WT and Scx-DTA mice

270 (Fig. 4A, D). A detailed section analysis confirmed that the gluteus maximus muscles

271 formed insertions with gluteus tuberosity through tendon cells at this stage, although the

272 tendon cells were reduced in Scx-DTA mice (Fig. 4B, C, E, F). As embryonic

273 development proceeds, the junction of the gluteus maximus muscle/tendon matured and

274 was firmly inserted into the gluteus tuberosity at E17.5 (Fig. 4G-I). On the other hand, the

275 tip of gluteus maximus muscle of $S c x$-DTA mice was distally dislocated while most of the

276 tendon and insertion into the gluteus tuberosity was lost by this stage (Fig. 4J-L). These

277 results imply that the skeletal muscle is able to reposition its attachment site according to

278 environmental changes, even after myofiber differentiation or the formation of initial

279 attachment. Recent studies also reported the post-natal elongation or post-fusion

280 repositioning of muscles (Gu et al., 2016; Huang et al., 2013), indicating that the

281 plasticity of muscle positioning is higher than generally considered. We assume that this

282 interaction between skeletal muscle and tendon provides musculoskeletal morphogenesis

283 reproducibility and robustness to resist environmental or genetic perturbations.

284 The molecular mechanism underlying this muscle-tendon interaction remains 
285 to be explored. In Scx-DTA mice, muscles in the zeugopod (i.e., the EDC/EDQ/ECU

286 muscles) fused and attached to the remaining tendons at their distal end (Fig. 2H). The

287 insertion sites of the muscles in the girdle (i.e., the pectoral and gluteus muscles)

288 dislocated toward the joint regions, such as the shoulder, elbow or knee (Fig. 2, Fig. S7B).

289 The remaining tendon cells were relatively abundant in the joint area of Scx-DTA

290 embryos (Fig. 1F, J, Fig. S7A), probably due to the initial amount and continuous cell

291 recruitment/differentiation from the $S c x$-negative cell population (Huang et al., 2019;

292 Shwartz et al., 2016). The fact that muscle did not attach randomly to nearby bone but

293 changed its morphology toward the distal tendon tissue implied a hypothesis that

294 diffusible molecule(s) secreted from tendon cells could attract myofibers. Indeed, recent

295 studies reported the involvement of retinoic acid in the formation of the extraocular

296 functional unit (Comai et al., 2020), and FGF or BMP signaling were active at the

297 interface of the embryonic tendon and muscle (Eloy-Trinquet, Wang, Edom-Vovard, \&

298 Duprez, 2009; Wang et al., 2010). However, cell adhesion molecule (Hasson et al., 2010)

299 or ECM (Kutchuk et al., 2015) can also play parallel roles. Moreover, LPM-derived cells

300 were recently suggested to convert their cell fate to the myogenic lineage at the tip of 
301 muscles and to regulate muscle patterning (de Lima et al., 2020). Clearly more studies are

302 required to fully understand the molecular and cellular mechanisms of precise skeletal

303 muscle patterning. We believe that revealing the molecular mechanism behind this

304 process would shed light on broad areas of biology, such as the diversity of muscle

305 patterning among species or regenerative medicine. 
308 Acknowledgements

309 We thank Drs. Scherer, Kist and Ohteki (TMDU) for providing the Sox9 flox and

310 Rosa26-LSL-DTA mice. This Research is supported by AMED-CREST from AMED

311 (JP20gm0810008), MEXT KAKENHI (19K06697), the Nakatomi Foundation, the

312 Nakajima Foundation and the Takeda Science Foundation for M.I.

315 Author Contributions

316 Y.O., T.S., C. S., and M.I. performed the experiments. H.A. and M.I. planned the study

317 and wrote the manuscript.

320 Declaration of Interests

321 The authors declare no conflicts of interest in association with the present study. 


\section{Methods}

\section{Mice}

325 A ScxCre-L transgenic ( $\mathrm{Tg}$ ) mouse strain has been described previously (Sugimoto,

326 Takimoto, Hiraki, \& Shukunami, 2013b). A Rosa26-LSL-DTA mouse strain was kindly

327 provide by Dr. Ohteki. The mouse was originally purchased from Jackson Laboratory

328 (B6.129P2-Gt(ROSA)26Sortm1(DTA)Lky/J, strain\#009669) by Dr. Ohteki and

329 transferred under the permission of Jackson Lab. A Mohawk-Venus knock-in mouse was

330 generated and described in our previous study (Ito et al., 2010). Myf5Cre

331 (B6.129S4-Myf5 $5^{\text {tm3(cre)Sor }} / J$, strain\#007893) (Tallquist, Weismann, Hellström, \& Soriano,

332 2000) and Meox2Cre mice $\left(B 6.129 S 4-M e o x 2^{\text {tml(cre)Sor }} / J\right.$, strain\#003755) (Tallquist \&

333 Soriano, 2000) were purchased from Jackson Laboratory. A Sox9-flox mouse strain was

334 kindly provided by Dr. Scherer and Dr. Kist (Kist, Schrewe, Balling, \& Scherer, 2002).

335 ICR mice were purchased from Sankyo lab-service (Tokyo, Japan). All animal

336 experiments were approved by the animal experiment committees of Meiji University

337 (approval No. IACUC17-0007) and the National Research Institute for Child Health and

338 Development (approval No. A2004-003). 


\section{Histological analyses}

341 For paraffin sections, embryos were fixed with $4 \%$ paraformaldehyde (PFA) for $4{ }^{\circ} \mathrm{C}$

342 overnight, dehydrated with methanol, cleared with xylene and embedded in paraffin

343 For cryosection, embryos were fixed with $4 \%$ PFA for $4^{\circ} \mathrm{C}$ overnight, washed with PBS

344 and embedded with OCT compound (Sakura Finetek, Osaka, Japan). The embryos were

345 sectioned at $7 \mu \mathrm{m}$ for hematoxylin and eosin (H\&E) staining, and TUNEL and

346 immunofluorescence analyses. The TUNEL analysis was performed using an In situ

347 Death Detection Kit (Roche, Basel, Switzerland) according to the manufacturer's

348 instruction. The names of muscles and tendons appeared in sections were judged

349 according to Watson et al. (Watson, Riordan, Pryce, \& Schweitzer, 2009).

\section{Immunohistochemistry}

352 The embryos were fixed with $4 \%$ PFA at $4{ }^{\circ} \mathrm{C}$ overnight, dehydrated with methanol and

353 rehydrated with PBS supplemented with 0.1\% triton-X100 (PBSTx). The embryos were

354 digested with $10 \mu \mathrm{g} / \mathrm{ml}$ Protease $\mathrm{K}$ at $37^{\circ} \mathrm{C}$ for $60 \mathrm{~min}$, re-fixed with $4 \%$ PFA, blocked 
355 with $2 \%$ BSA/PBSTx and incubated with $\alpha$-MHC antibody (Sigma-Aldrich, St. Louis,

356 USA, My-32, 1:1000 in $2 \%$ BSA/PBSTx) at $4^{\circ} \mathrm{C}$ overnight. Then the embryos were

357 washed 10 times with PBSTx, incubated with $\alpha$-mouse IgG-AP conjugated (1:2000 in

358 2\% BSA/PBSTx) (ab5880 Abcam, Cambridge, UK) at $4^{\circ} \mathrm{C}$ overnight, washed 10 times

359 with PBSTx and the signal was developed in NBT/BCIP solution (Roche).

361 Immunofluorescence

362 Paraffin or cryosections were prepared as described in the histology section, boiled in

363 citric acid ( $\mathrm{pH} 2.0)$ for antigen retrieval and stained with an $\alpha-\mathrm{MHC}$ antibody (Sigma

364 My-32, 1:1000) or $\alpha$-GFP antibody (Abcam ab13970, 1:1000). $\alpha$-mouse IgG-Cy3

365 (Jackson ImmunoResearch, West Grove, USA, 1:1000) or $\alpha$-chicken IgG-Alexa488

366 (Thermo Fisher, Waltham, USA, 1:1000) were used as secondary antibodies.

\section{Whole mount in situ hybridization}

369 Whole mount in situ hybridization (WISH) was performed according to the methods of

370 Yokoyama et al. (Yokoyama et al., 2009). Briefly, the embryos were fixed with 4\% PFA 
371 at $4^{\circ} \mathrm{C}$ overnight, dehydrated with methanol and rehydrated with PBS supplemented

372 with $0.1 \%$ tween20 (PBST). The embryos were digested with $10 \mu \mathrm{g} / \mathrm{ml}$ Protease $\mathrm{K}$ at

$37337^{\circ} \mathrm{C}$ for $20-60$ min (depending on the stage), re-fixed with $4 \%$ PFA, and hybridized

374 with an anti-sense probe labeled with digoxigenin (DIG) or fluorescein in hybridization

375 buffer at $65^{\circ} \mathrm{C}$ overnight. The embryos were washed with wash buffer at $65^{\circ} \mathrm{C}$, blocked

376 with $10 \%$ FBS/PBST for 2 hours, and incubated with $\alpha$-DIG or $\alpha$-fluorescein antibody

377 conjugated with alkaline phosphatase (Roche, 1:2000 in $10 \% \mathrm{FBS} / \mathrm{PBST}$ ) at $4^{\circ} \mathrm{C}$

378 overnight. Then the embryos were washed 10 times with PBST and the signal was

379 developed in NBT/BCIP solution (Roche). For double in situ hybridization,

380 DIG-labeled tnmd probe and fluorescein-labeled myh3 probe were simultaneously

381 hybridized. Tnmd was stained with $\alpha$-DIG-AP antibody and NBT/BCIP substrate,

382 post-fixed with 4\% PFA, dehydrated with methanol, rehydrated with PBST and myh3

383 was stained with $\alpha$-fluorescein-AP antibody and INT (Roche)/BCIP (Roche) substrates.

384 The sequences of the primers used for the amplification of probe are as follows:

385 myogenin fw: 5'- ACCTGATGGAGCTGTATGAGACATC -3', myogenin rev: 5'-

386 CATTTAGGTGACACTATAGCAGATGTGCACACTTGTCCAGG -3', myh3 fw: 5'- 
387 CGTTTTGGACATTGCGGGTT -3', myh3 rev: 5'- ATGGACTCCCTCCTCTGCAT

$388-3^{\prime}$.

389

\section{Skeletal preparation}

391 The embryos were removed with their skin and internal organs, fixed with $100 \%$

392 ethanol, and serially stained with $0.03 \%$ alcian blue (Sigma-Aldrich) solution and

$3930.01 \%$ alizarin red (Sigma-Aldrich) solution. When embryos were used after

394 whole-mount IHC, the embryos were post-fixed with $4 \%$ PFA, dehydrated with ethanol

395 and stained as described above. 


\section{References}

Asahara, H., Inui, M., \& Lotz, M. K. (2017). Tendons and Ligaments: Connecting Developmental Biology to Musculoskeletal Disease Pathogenesis. Journal of Bone and Mineral Research : the Official Journal of the American Society for Bone and Mineral Research, 6(2), 181-10. http://doi.org/10.1002/jbmr.3199

Besse, L., Sheeba, C. J., Holt, M., Labuhn, M., Wilde, S., Feneck, E., et al. (2020). Individual Limb Muscle Bundles Are Formed through Progressive Steps Orchestrated by Adjacent Connective Tissue Cells during Primary Myogenesis. CellReports, 30(10), 3552-3565.e6. http://doi.org/10.1016/j.celrep.2020.02.037

Blitz, E., Sharir, A., Akiyama, H., \& Zelzer, E. (2013). Tendon-bone attachment unit is formed modularly by a distinct pool of Scx- and Sox9-positive progenitors. Development (Cambridge, England), 140(13), 2680-2690. http://doi.org/10.1242/dev.093906

Braun, T., Rudnicki, M. A., Arnold, H. H., \& Jaenisch, R. (1992). Targeted inactivation of the muscle regulatory gene Myf-5 results in abnormal rib development and perinatal death. Cell, 71(3), 369-382.

Brent, A. E., Braun, T., \& Tabin, C. J. (2005). Genetic analysis of interactions between the somitic muscle, cartilage and tendon cell lineages during mouse development. Development (Cambridge, England), 132(3), 515-528. http://doi.org/10.1242/dev.01605

Buckingham, M., \& Rigby, P. W. J. (2014). Gene regulatory networks and transcriptional mechanisms that control myogenesis. Developmental Cell, 28(3), 225-238. http://doi.org/10.1016/j.devcel.2013.12.020

Buckingham, M., Bajard, L., Chang, T., Daubas, P., Hadchouel, J., Meilhac, S., et al. (2003). The formation of skeletal muscle: from somite to limb. Journal of Anatomy, 202(1), 59-68.

Comai, G., \& Tajbakhsh, S. (2014). Molecular and cellular regulation of skeletal myogenesis. Current Topics in Developmental Biology, 110, 1-73. http://doi.org/10.1016/B978-0-12-405943-6.00001-4

Comai, G., Sambasivan, R., Gopalakrishnan, S., \& Tajbakhsh, S. (2014). Variations in the efficiency of lineage marking and ablation confound distinctions between myogenic cell populations. Developmental Cell, 31(5), 654-667. 
Comai, G., Tesarova, M., Dupé, V., Rhinn, M., Vallecillo-García, P., da Silva, F., et al. (2020). Local retinoic acid directs emergence of the extraocular muscle functional unit. bioRxiv, 107(Pt 3), 126-48. http://doi.org/10.1101/2020.01.07.897694

de Lima, J. E., Blavet, C., Bonnin, M.-A., Hirsinger, E., Comai, G., Yvernogeau, L., et al. (2020). BMP signalling directs a fibroblast-to-myoblast conversion at the connective tissue/muscle interface to pattern limb muscles. bioRxiv, 41, 2020.07.20.211342. http://doi.org/10.1101/2020.07.20.211342

Dietrich, S., Abou-Rebyeh, F., Brohmann, H., Bladt, F., Sonnenberg-Riethmacher, E., Yamaai, T., et al. (1999). The role of SF/HGF and c-Met in the development of skeletal muscle. Development (Cambridge, England), 126(8), 1621-1629.

Eloy-Trinquet, S., Wang, H., Edom-Vovard, F., \& Duprez, D. (2009). Fgf signaling components are associated with muscles and tendons during limb development. Developmental Dynamics, 238(5), 1195-1206. http://doi.org/10.1002/dvdy.21946

Griffin, C. A., Apponi, L. H., Long, K. K., \& Pavlath, G. K. (2010). Chemokine expression and control of muscle cell migration during myogenesis. Journal of Cell Science, 123(Pt 18), 3052-3060. http://doi.org/10.1242/jcs.066241

Gu, J.-M., Wang, D. J., Peterson, J. M., Shintaku, J., Liyanarachchi, S., Coppola, V., et al. (2016). An NF-кB - EphrinA5-Dependent Communication between NG2(+) Interstitial Cells and Myoblasts Promotes Muscle Growth in Neonates. Developmental Cell, 36(2), 215-224. http://doi.org/10.1016/j.devcel.2015.12.018

Guerquin, M.-J., Charvet, B., Nourissat, G., Havis, E., Ronsin, O., Bonnin, M.-A., et al. (2013). Transcription factor EGR1 directs tendon differentiation and promotes tendon repair. Journal of Clinical Investigation, 123(8), 3564-3576. http://doi.org/10.1172/JCI67521

Guha, U., Gomes, W. A., Kobayashi, T., Pestell, R. G., \& Kessler, J. A. (2002). In vivo evidence that BMP signaling is necessary for apoptosis in the mouse limb. Developmental Biology, 249(1), 108-120. http://doi.org/10.1006/dbio.2002.0752 Hasson, P., DeLaurier, A., Bennett, M., Grigorieva, E., Naiche, L. A., Papaioannou, V. E., et al. (2010). Tbx4 and Tbx5 Acting in Connective TissueAre Required for Limb Muscle and Tendon Patterning. Devcel, 18(1), 148-156. http://doi.org/10.1016/j.devcel.2009.11.013

Helmbacher, F., \& Stricker, S. (2020). Tissue cross talks governing limb muscle 
development and regeneration. Seminars in Cell \& Developmental Biology, 104, 14-30. http://doi.org/10.1016/j.semcdb.2020.05.005

Huang, A. H., Riordan, T. J., Pryce, B., Weibel, J. L., Watson, S. S., Long, F., et al. (2015). Musculoskeletal integration at the wrist underlies the modular development of limb tendons. Development (Cambridge, England), 142(14), 2431-2441.

Huang, A. H., Riordan, T. J., Wang, L., Eyal, S., Zelzer, E., Brigande, J. V., \& Schweitzer, R. (2013). Repositioning forelimb superficialis muscles: tendon attachment and muscle activity enable active relocation of functional myofibers. Developmental Cell, 26(5), 544-551. http://doi.org/10.1016/j.devcel.2013.08.007 Huang, A. H., Watson, S. S., Wang, L., Baker, B. M., Akiyama, H., Brigande, J. V., \& Schweitzer, R. (2019). Requirement for scleraxis in the recruitment of mesenchymal progenitors during embryonic tendon elongation. Development (Cambridge, England), 146(20). http://doi.org/10.1242/dev.182782

Ito, Y., Toriuchi, N., Yoshitaka, T., Ueno-Kudoh, H., Sato, T., Yokoyama, S., et al. (2010). The Mohawk homeobox gene is a critical regulator of tendon differentiation. Proceedings of the National Academy of Sciences, 107(23), 10538-10542. http://doi.org/10.1073/pnas.1000525107/-/DCSupplemental/pnas.201000525SI.pdf

Kague, E., Hughes, S. M., Lawrence, E. A., Cross, S., Martin-Silverstone, E., Hammond, C. L., \& Hinits, Y. (2019). Scleraxis genes are required for normal musculoskeletal development and for rib growth and mineralization in zebrafish. The FASEB Journal : Official Publication of the Federation of American Societies for Experimental Biology, 33(8), 9116-9130. http://doi.org/10.1096/fj.201802654RR

Kardon, G. (1998). Muscle and tendon morphogenesis in the avian hind limb. Development (Cambridge, England), 125(20), 4019-4032.

Kardon, G. (2011). Development of the musculoskeletal system: meeting the neighbors. Development (Cambridge, England), 138(14), 2855-2859. http://doi.org/10.1242/dev.067181

Kardon, G., Harfe, B. D., \& Tabin, C. J. (2003). A Tcf4-positive mesodermal population provides a prepattern for vertebrate limb muscle patterning. Devcel, 5(6), 937-944. http://doi.org/10.1016/s1534-5807(03)00360-5

Kist, R., Schrewe, H., Balling, R., \& Scherer, G. (2002). Conditional inactivation of 
495

496

497

498

499

500

501

502

503

504

505

506

507

508

509

510

511

512

513

514

515

516

517

518

519

520

521

522

523

524

525

526

527

Sox9: a mouse model for campomelic dysplasia. Genesis (New York, NY : 2000), 32(2), 121-123. http://doi.org/10.1002/gene.10050

Kozhemyakina, E., Lassar, A. B., \& Zelzer, E. (2015). A pathway to bone: signaling molecules and transcription factors involved in chondrocyte development and maturation. Development (Cambridge, England), 142(5), 817-831. http://doi.org/10.1242/dev.105536

Kutchuk, L., Laitala, A., Soueid-Bomgarten, S., Shentzer, P., Rosendahl, A. H., Eilot, S., et al. (2015). Muscle composition is regulated by a Lox-TGF feedback loop. Development (Cambridge, England), 142(5), 983-993. http://doi.org/10.1242/dev.113449

Li, Y., Qiu, Q., Watson, S. S., Schweitzer, R., \& Johnson, R. L. (2010). Uncoupling skeletal and connective tissue patterning: conditional deletion in cartilage progenitors reveals cell-autonomous requirements for Lmx1b in dorsal-ventral limb patterning. Development (Cambridge, England), 137(7), 1181-1188. http://doi.org/10.1242/dev.045237

Murchison, N. D., Price, B. A., Conner, D. A., Keene, D. R., Olson, E. N., Tabin, C. J., \& Schweitzer, R. (2007). Regulation of tendon differentiation by scleraxis distinguishes force-transmitting tendons from muscle-anchoring tendons. Development (Cambridge, England), 134(14), 2697-2708.

$$
\text { http://doi.org/10.1242/dev.001933 }
$$

Pryce, B. A., Watson, S. S., Murchison, N. D., Staverosky, J. A., Dunker, N., \& Schweitzer, R. (2009). Recruitment and maintenance of tendon progenitors by TGF signaling are essential for tendon formation. Development (Cambridge, England), 136(8), 1351-1361. http://doi.org/10.1242/dev.027342

Rodriguez-Guzman, M., Montero, J. A., Santesteban, E., Gañan, Y., Macias, D., \& Hurle, J. M. (2007a). Tendon-muscle crosstalk controls muscle bellies morphogenesis, which is mediated by cell death and retinoic acid signaling. Developmental Biology, 302(1), 267-280. http://doi.org/10.1016/j.ydbio.2006.09.034

Rodriguez-Guzman, M., Montero, J. A., Santesteban, E., Gañan, Y., Macias, D., \& Hurle, J. M. (2007b). Tendon-muscle crosstalk controls muscle bellies morphogenesis, which is mediated by cell death and retinoic acid signaling. Developmental Biology, 302(1), 267-280. 
Schweitzer, R., Zelzer, E., \& Volk, T. (2010). Connecting muscles to tendons: tendons and musculoskeletal development in flies and vertebrates. Development (Cambridge, England), 137(17), 2807-2817. http://doi.org/10.1242/dev.047498

Shukunami, C., Oshima, Y., \& Hiraki, Y. (2001). Molecular cloning of tenomodulin, a novel chondromodulin-I related gene. Biochemical and Biophysical Research Communications, 280(5), 1323-1327. http://doi.org/10.1006/bbrc.2001.4271

Shukunami, C., Takimoto, A., Nishizaki, Y., Yoshimoto, Y., Tanaka, S., Miura, S., et al. (2018). Scleraxis is a transcriptional activator that regulates the expression of Tenomodulin, a marker of mature tenocytes and ligamentocytes. Scientific Reports, 8(1), 3155-17. http://doi.org/10.1038/s41598-018-21194-3

Shwartz, Y., Viukov, S., Krief, S., \& Zelzer, E. (2016). Joint Development Involves a Continuous Influx of Gdf5-Positive Cells. CellReports, 15(12), 2577-2587. http://doi.org/10.1016/j.celrep.2016.05.055

Sugimoto, Y., Takimoto, A., Akiyama, H., Kist, R., Scherer, G., Nakamura, T., et al. (2013a). Scx+/Sox9+ progenitors contribute to the establishment of the junction between cartilage and tendon/ligament. Development (Cambridge, England), 140(11), 2280-2288. http://doi.org/10.1242/dev.096354

Sugimoto, Y., Takimoto, A., Hiraki, Y., \& Shukunami, C. (2013b). Generation and characterization of ScxCre transgenic mice. Genesis (New York, NY : 2000), 51(4), 275-283. http://doi.org/10.1002/dvg.22372

Swinehart, I. T., Schlientz, A. J., Quintanilla, C. A., Mortlock, D. P., \& Wellik, D. M. (2013). Hox11 genes are required for regional patterning and integration of muscle, tendon and bone. Development (Cambridge, England), 140(22), 4574-4582.

$$
\text { http://doi.org/10.1242/dev.096693 }
$$

Tallquist, M. D., \& Soriano, P. (2000). Epiblast-restricted Cre expression in MORE mice: a tool to distinguish embryonic vs. extra-embryonic gene function. Genesis (New York, NY : 2000), 26(2), 113-115.

Tallquist, M. D., Weismann, K. E., Hellström, M., \& Soriano, P. (2000). Early

$$
\text { myotome specification regulates PDGFA expression and axial skeleton }
$$
development. Development (Cambridge, England), 127(23), 5059-5070.

Vallecillo-García, P., Orgeur, M., Hofe-Schneider, vom, S., Stumm, J., Kappert, V., Ibrahim, D. M., et al. (2017). Odd skipped-related 1 identifies a population of 
561 embryonic fibro-adipogenic progenitors regulating myogenesis during limb

562 development. Nature Communications, 8(1), 1218-18.

563 http://doi.org/10.1038/s41467-017-01120-3

564 Voehringer, D., Liang, H.-E., \& Locksley, R. M. (2008). Homeostasis and effector

565 function of lymphopenia-induced "memory-like" $\mathrm{T}$ cells in constitutively $\mathrm{T}$

566 cell-depleted mice. Journal of Immunology (Baltimore, Md : 1950), 180(7),

$567 \quad 4742-4753$.

568 Wang, H., Noulet, F., Edom-Vovard, F., Tozer, S., Le Grand, F., \& Duprez, D. (2010).

569 Bmp signaling at the tips of skeletal muscles regulates the number of fetal muscle

570 progenitors and satellite cells during development. Developmental Cell, 18(4),

571 643-654. http://doi.org/10.1016/j.devcel.2010.02.008

572 Watson, S. S., Riordan, T. J., Pryce, B. A., \& Schweitzer, R. (2009). Tendons and

573 muscles of the mouse forelimb during embryonic development. Developmental

574 Dynamics, 238(3), 693-700. http://doi.org/10.1002/dvdy.21866

575 Yokoyama, S., Ito, Y., Ueno-Kudoh, H., Shimizu, H., Uchibe, K., Albini, S., et al.

576 (2009). A systems approach reveals that the myogenesis genome network is

577 regulated by the transcriptional repressor RP58. Developmental Cell, 17(6),

578 836-848. http://doi.org/10.1016/j.devcel.2009.10.011

579

580

581 


\section{$582 \quad$ Figure legends}

583 Figure 1 Tendon and ligament tissues are reduced in Scx-DTA mice.

584 (A) A schematic drawing showing the generation of the Scx-DTA mouse. Illustrations

585 are provided by (C2016 DBCLS TogoTV. (B-E) H\&E staining (B, D) and the TUNEL

586 analysis (C, E) of the E15.5 embryonic tail. The blue arrowhead indicates the normal

587 tail tendon and the red arrowhead indicates ablated tendon tissue. Scalebar: $100 \mu \mathrm{m}$.

588 (F-M) The fluorescence signal from Mkx-Venus (MkxVen) knock-in reporter visualized

589 the tendon tissue in E17.5 embryos. The blue arrowhead indicates normal limb tendons

590 and the red arrowhead indicates ablated tendon tissue. Scalebar: $100 \mu \mathrm{m} .(\mathrm{N}-\mathrm{U}) \mathrm{H} \& \mathrm{E}$

591 staining of E15.5 embryonic tendon/ligament tissues. Ach. tendon, Achilles' tendon;

592 Cruc. Ligament, cruciate ligament; Intervert. Disc., intervertebral disc. Scalebar: 100

$593 \mu \mathrm{m}$. (V) A table summarizing the genotypes of obtained P1 pups from crossing of

594 ScxCre-L Tg and Rosa26-LSL-DTA. (W) The gross appearance of P0 pups. A Scx-DTA

595 pup is shown on the left and a control pup is shown on the right. Scx-DTA pups were

596 pale and the milk spot was not observed. (Y) Alcian-blue and alizarin-red staining

597 showing the skeletal elements of P0 pups. An Scx-DTA pup is shown on the left and a 
598 control pup is shown on the right. The blue arrowhead indicates the normal ribcage and

599 the red arrowhead indicates the reduced ribcage. For all the experiments, at least three

600 embryos were examined $(n>3)$ and consistent results were obtained. The figures show

601 representative results.

602

603

604

605 
606 Figure 2

607 The muscle shape and position were altered in the Scx-DTA mouse.

608 (A-H) Whole-mount immunohistochemistry of myosin heavy chain (MHC) in forelimbs

609 of P0 pups. Del.: Deltoid muscle. The blue arrowhead in C indicates the normal

610 insertion site of the deltoid muscle (deltoid tuberosity). The red arrowhead in G

611 indicates an altered insertion site of the deltoid (shoulder joint). The arrows in D and $\mathrm{H}$

612 indicate the extensor pollicis muscles. The blue arrowheads in D indicate separated

613 ECU and EDQ muscles. The red arrowhead in $\mathrm{H}$ indicates fused ECU/EDQ muscle.

614 (I-O) Immunofluorescence of MHC and MkxVen (using $\alpha$-EGFP) in the forelimbs of an

615 E18.5 embryo. The blue arrowhead in J' indicates FDS tendons. The red arrowhead in

616 M' indicates distally dislocated FDS muscle. The blue arrowhead in K indicates FDS

617 muscles that are missing in the Scx-DTA mouse (red arrowhead in N). The blue arrow in

$618 \mathrm{~K}$ indicates normal extensor carpi muscles in a control embryo. The red arrow in $\mathrm{N}$

619 indicates rearranged extensor carpi muscles in an Scx-DTA embryo. The photograph in

$620 \mathrm{O}$ shows the position of sections I-N. Scalebar: $100 \mu \mathrm{m}$. (P-S) Whole-mount

621 immunohistochemistry of MHC in E17.5 embryos. The blue arrowhead in P' indicates 
622 the normal insertion site of the pectoralis major muscle (deltoid tuberosity). The red

623 arrowhead in R' indicates a dislocated insertion site of the pectoralis major muscle

624 (elbow joint). The blue arrowhead in Q' indicates the normal insertion site of the gluteus

625 maximus muscle (gluteus tuberosity). The red arrowhead in $S^{\prime}$ 'indicates a dislocated

626 insertion site of the gluteus major muscle (knee joint). (T-W) Whole-mount in situ

627 hybridization of $m y h 3$ in the rectus abdominus (T, V, Rec. Abdomin.) and diaphragm (U,

$628 \mathrm{~W})$. The blue arrowheads in $\mathrm{T}$ and $\mathrm{U}$ indicate the normal organization of abdominal

629 muscles. The red arrowheads in $\mathrm{V}$ and $\mathrm{W}$ indicate a disorganized pattern of abdominal

630 muscles. The arrows in $\mathrm{U}$ and $\mathrm{W}$ indicate the width of the central tendons. For all of the

631 experiments, at least three pups/embryos were examined $(n>3)$ and consistent results

632 were obtained. The figures show representative results.

633

634 
635 Figure 3

636 Loss of the tendon cells affects the patterning of myofibers.

637 (A-F) A whole-mount in situ hybridization (WISH) analysis visualized the myoblast

638 localization. The blue arrowheads in $\mathrm{A}$ and $\mathrm{D}$ indicate the normal migration of

639 Pax3-positive myoblasts into the E11.5 limb. The blue arrowheads in B and E indicate

640 the normal segregation of Myog-positive myoblasts in the E12.5 limb. The blue

641 arrowheads in $\mathrm{C}$ and $\mathrm{F}$ indicate normal positioning of Myog-positive myoblasts in the

642 E13.5 limb. (G-L) A WISH analysis visualizes myofiber patterning. The blue

643 arrowheads in $\mathrm{G}$ and $\mathrm{J}$ indicate the normal location of $M y h 3$-positive myofibers in the

644 E12.5 limb. The blue arrowheads in $\mathrm{H}$ and I indicate the normal boundaries of the

645 Myh3-positive FDC muscle bundle in E13.5 and E14.5 limbs. The red arrowheads in K

646 and L indicate obscure or fused boundaries of the Myh3-positive FDC muscle bundle in

647 E13.5 and E14.5 limbs. (M-P) Whole-mount immunohistochemistry visualized myosin

648 heavy chain (MHC)-positive myofibers in E14.5 embryos. The blue arrowheads in M

649 and $\mathrm{N}$ indicate the normal insertion site of the deltoid muscle (deltoid tuberosity). The

650 red arrowheads in $\mathrm{O}$ and $\mathrm{P}$ indicate a dislocated insertion site of the deltoid muscle 
bioRxiv preprint doi: https://doi.org/10.1101/2021.01.03.424463; this version posted January 4, 2021. The copyright holder for this preprint (which was not certified by peer review) is the author/funder, who has granted bioRxiv a license to display the preprint in perpetuity. It is made available under aCC-BY-NC-ND 4.0 International license.

651 (shoulder joint). At least three embryos were examined in all the experiments $(n>3)$ and

652 consistent results were obtained. The figures show representative results 
654 Figure 4

\section{The attachment site of muscle was repositioned after myofiber differentiation.}

656 (A, D) Whole-mount immunohistochemistry visualized myosin heavy chain

657 (MHC)-positive myofibers in the hind limb of E14.5 embryos. Dotted lines indicate the

658 position of the section shown in B, C, E, and F. Arrowheads indicate the position of the

659 gluteus tuberosities. (B, C, E, F) The immunofluorescence analysis of the E14.5

660 hindlimb. Arrowheads indicate the position of the gluteus tuberosities. Scalebar: $100 \mu \mathrm{m}$.

661 (G, J) Whole-mount immunohistochemistry visualized myosin heavy chain

662 (MHC)-positive myofibers in the hind limb of E17.5 embryos. Dotted lines indicate the

663 position of the section shown in $\mathrm{H}, \mathrm{I}, \mathrm{K}$, and $\mathrm{L}$. The blue arrowhead in $\mathrm{G}$ indicates the

664 normal insertion site of the gluteus maximus muscle (gluteus tuberosity). The red

665 arrowhead in $\mathrm{J}$ indicates the dislocated insertion site of the gluteus maximus muscle

666 (knee joint). (H, I, K, L) The immunofluorescence analysis of the E17.5 hindlimb.

667 Arrowheads indicate the position of gluteus tuberosities. Scalebar: $100 \mu \mathrm{m}$. In all of the

668 experiments, at least three embryos were examined $(n>3)$ and consistent results were

669 obtained. The figures show representative results 
$671 \quad$ Figure S1

672 Tendon/ligament-specific cell death was observed in the Scx-DTA mouse.

673 (A-D) A TUNEL analysis of the E15.5 forelimb. (E-H) A TUNEL analysis of the E12.5

674 forelimb and shoulder. (I-P) H\&E staining and a TUNEL analysis of the E12.5 vertebrae

675 and tail.

676

677 Figure S2

678 The deltoid tuberosity is missing in the Scx-DTA mouse.

679 (A, B) Alcian-blue and alizarin-red staining of P0 pups. The blue arrowhead indicates

680 the normal deltoid tuberosity in a control limb (A), which is missing in the Scx-DTA

681 forelimb (B, red arrowhead)

682

683 Figure $\mathrm{S} 3$

684 Muscle patterning was not affected in the hindlimb of the Scx-DTA mouse.

685 (A, C) Whole-mount immunohistochemistry visualized MHC-positive myofibers in the

686 P0 hindlimb. (B, D) An immunofluorescence analysis of MHC in an E15.5 hindlimb 
section. Scale bar: $100 \mu \mathrm{m}$

688

689 Figure S4

690 The MCT gene expression was not changed in the Scx-DTA mouse.

691 Quantitative RT-PCR showed a significant reduction in the expression of tendon genes

692 in the E14.5 Scx-DTA forelimb; however, the expression of MCT genes was not

693 changed. Student's t-test was performed. $* \mathrm{p}<0.05$, **p $<0.01$, n.s.: no significant

694 difference.

695

696 Figure S5

697 The high expression of Myh3 marks the muscle-tendon junction.

698 A whole-mount in situ hybridization (WISH) analysis visualized the Myh3 and Tnmd

699 expression in the E14.5 hindlimbs. In A and D, Myh3 was stained with INT/BCIP

700 (orange) and Tnmd was stained with NBT/BCIP (blue). Arrowheads indicate the

701 putative junction between the EDL muscle and the EDL tendon. 
703 Figure S6

\section{Skeletal malformation did not affect muscle patterning.}

705 (A, C) Whole-mount immunohistochemistry visualized MHC-positive myofibers in

706 E16.5 pectoral muscles. The blue arrowheads indicate the normal insertion site of the

707 pectoralis major muscles (deltoid tuberosity) in control and Sox9 heterozygous embryos.

708 (B, D) Alcian-blue and alizarin-red staining showing the skeletal elements of embryos

709 shown in A and $\mathrm{C}$. The blue arrowhead indicates the normal deltoid tuberosity in a

710 control limb (B), which is missing in the Sox9 heterozygous forelimb (D, red

711 arrowhead). (E, G) Whole-mount immunohistochemistry visualized MHC-positive

712 myofibers in the E16.5 pectoral muscles. The blue arrowheads indicate the normal

713 insertion site of the pectoralis major muscles (deltoid tuberosity) in control and

$714 M y f 5^{\text {Cre/Cre }}$ embryos. (F, H) Alcian-blue and alizarin-red staining showing the skeletal

715 elements of embryos shown in E and G. The blue arrowhead indicates a normal rib cage

716 in a control embryo (E), which is diminished in $M y f 5^{\text {Cre/Cre }}$ embryo (H, red arrowhead).

717 (I, J) Whole-mount immunohistochemistry visualized MHC-positive myofibers in the 
718 E16.5 pectoral muscles. The blue arrowheads indicate the normal insertion site of the

719 pectoralis major muscles (deltoid tuberosity) in control and Myf5-DTA embryos.

$721 \quad$ Figure $\mathrm{S} 7$

722 Dislocation of the insertion site toward the joint region where tendon cells are

\section{3 abundant.}

724 (A) The immunofluorescence analysis of MkxVen (using $\alpha$-EGFP) in the E17.5 femur

725 of control (upper panel) and Scx-DTA (lower panel) embryos. The blue arrowheads

726 indicate the proximal and distal tendons for the gluteus major muscle in the control

727 embryo, which are missing in the $S c x$-DTA embryo (red arrowhead). Yellow arrowheads

728 indicate a comparable amount of tendon tissue in the knee joint of control and Scx-DTA

729 embryos. (B) Whole-mount in situ hybridization of $m y h 3$ in the E14.5 forelimb of

730 control and Scx-DTA embryos. The blue arrowheads indicate the normal attachment

731 sites of limb muscles. The red arrowhead indicates the accumulation of attachment sites

732 to the shoulder joint. 
bioRxiv preprint doi: https://doi.org/10.1101/2021.01.03.424463; this version posted January 4,2021 . The copyright holder for this preprint (which was not certified by peer review) is the author/funder, who has granted bioRxiv a license to display the preprint in perpetuity. It is made available under aCC-BY-NC-ND 4.0 International license.

734 
bioRxiv preprint doi: https://doi.org/10.1101/2021.01.03.424463; this version posted Januarv 4. 2021. The copvriaht holder for this preprint (which was not certified by peer review) is the author/funder, who has granted bioRxiv a license to display the preprint in perpetuity. It is made available under aCC-BY-NC-ND 4.0 International license.

735 


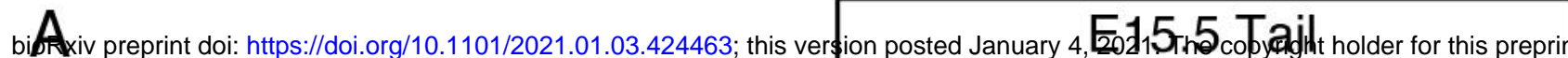
(which was not certified by peer review) is the author/funder, who has gran ScxCreL-Tg;

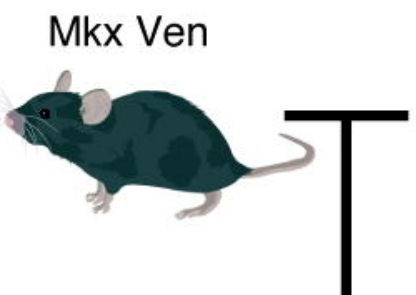

Tendon cell ablated

Embryos Rosa26-LSL-DTA
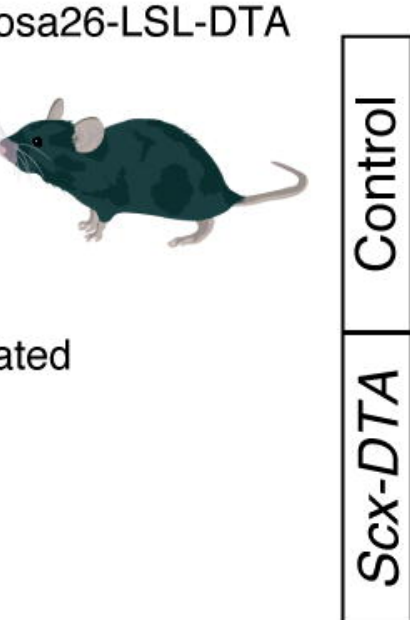

TUNEL

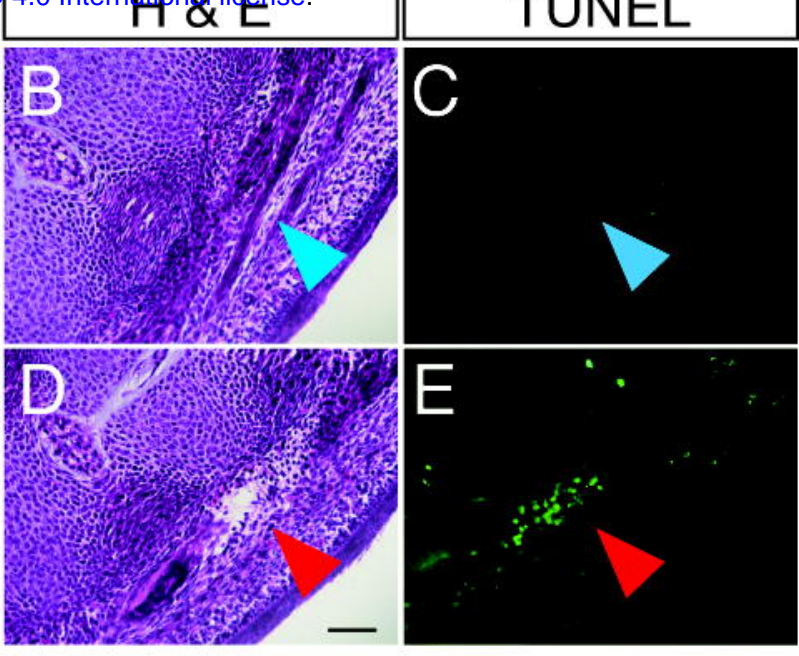

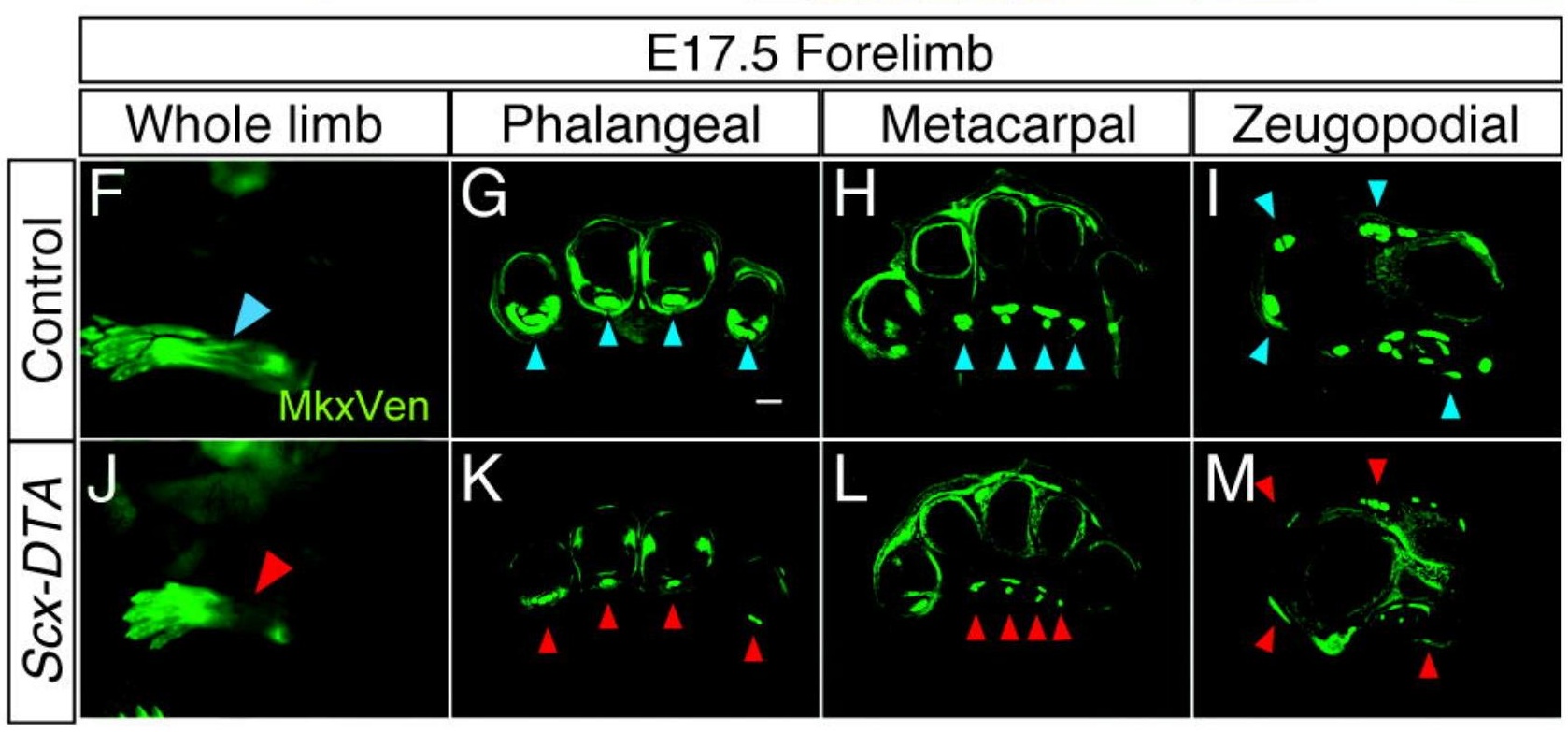

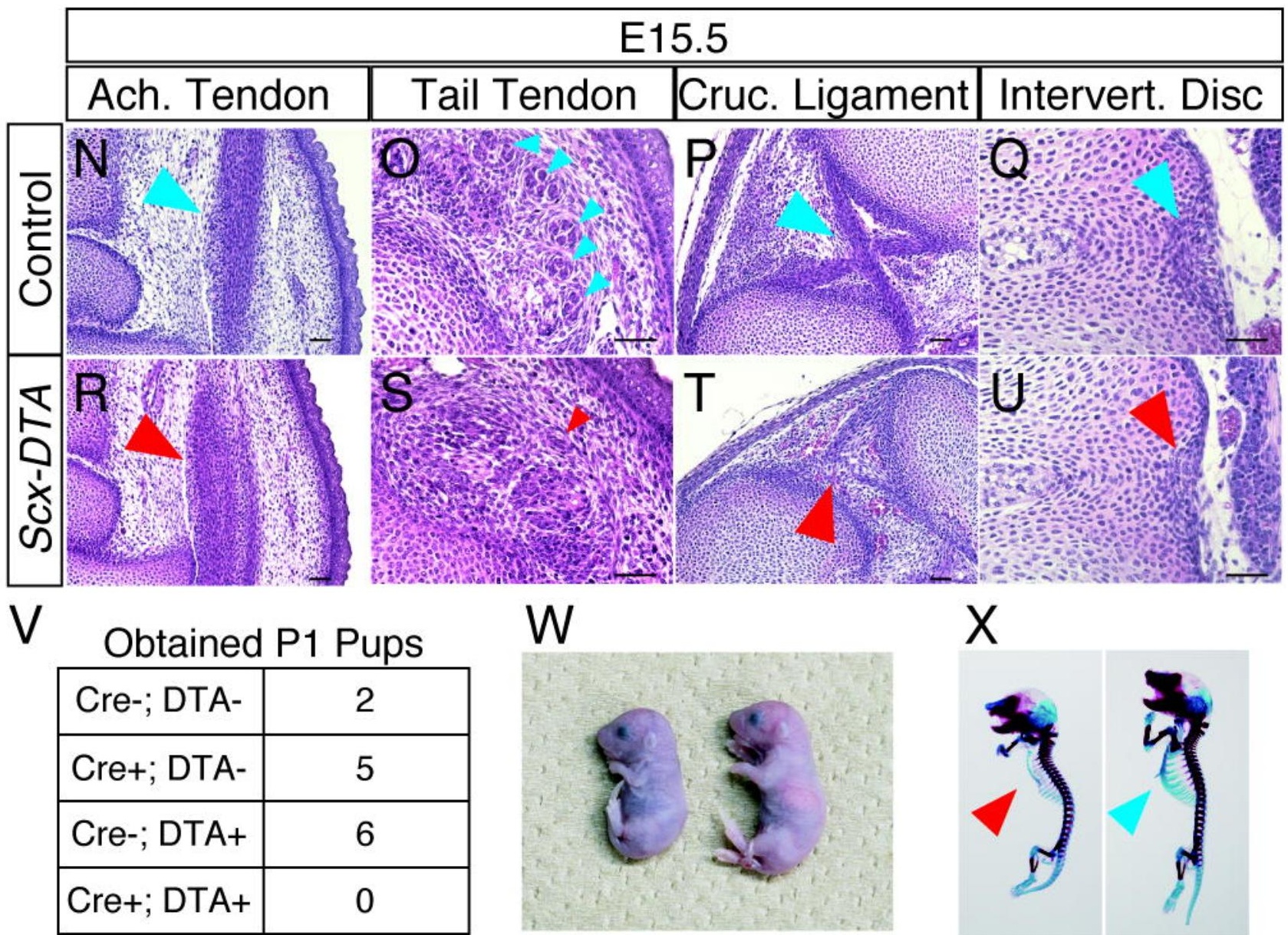


Ono et al. Figure 2
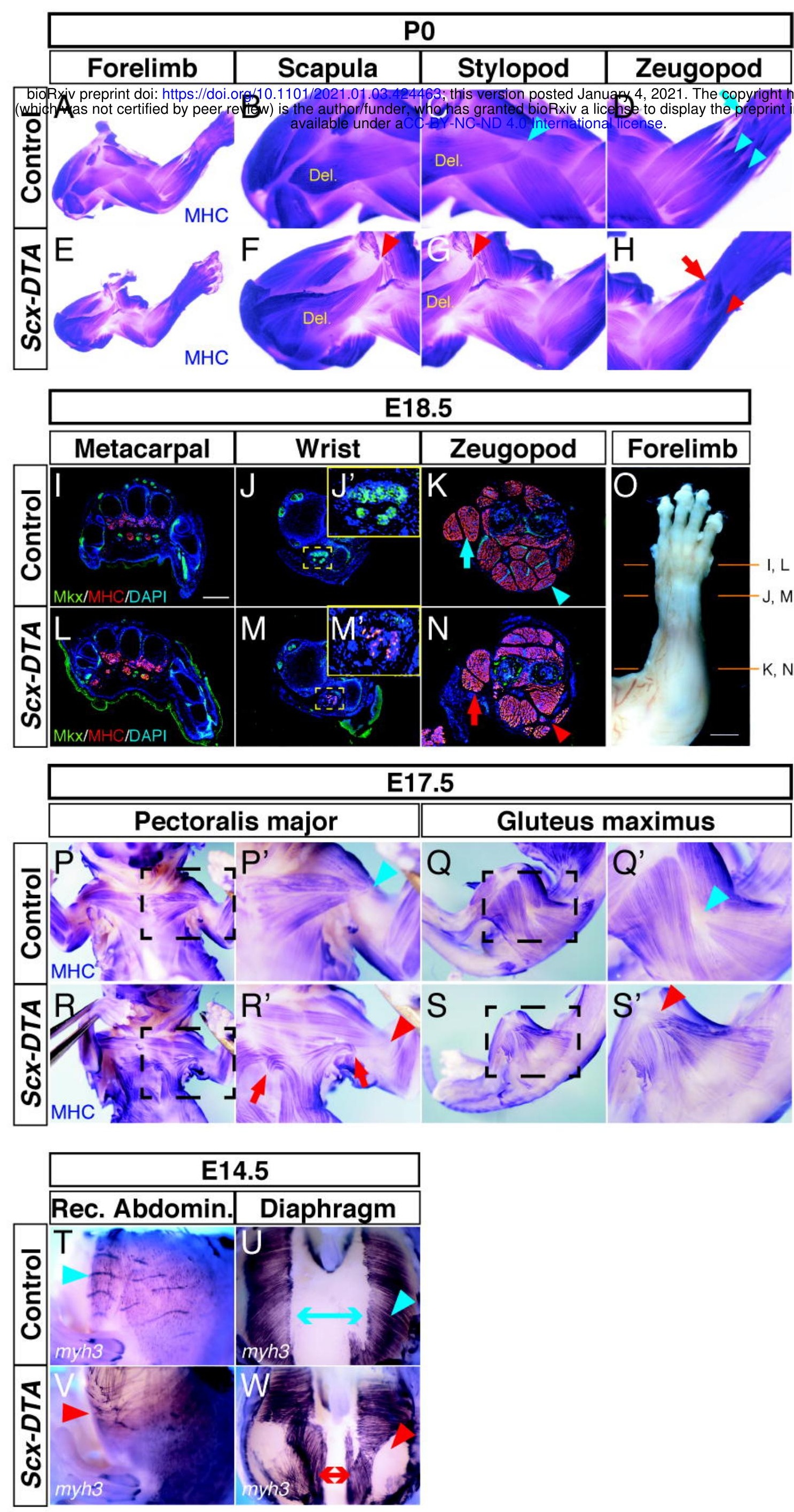
Ono et al. Figure 4

\section{E14.5 Gluteus maximus}

\section{Wholemount Gluteus tuberosity}
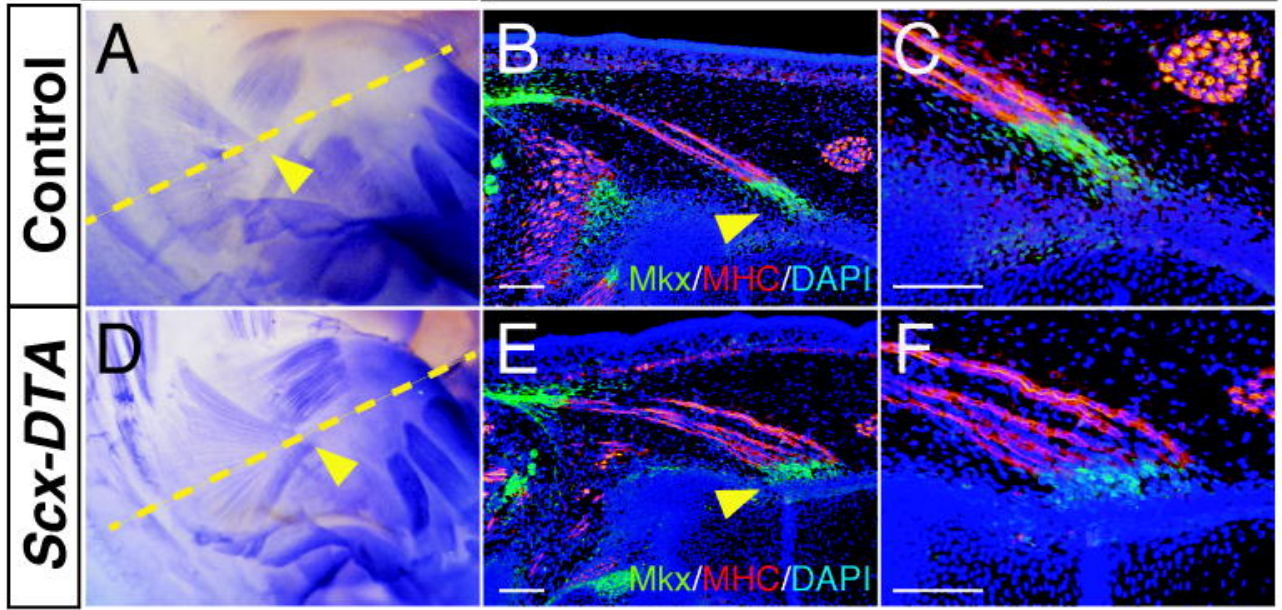

\section{E17.5 Gluteus maximus}

\section{Wholemount \\ Gluteus tuberosity}
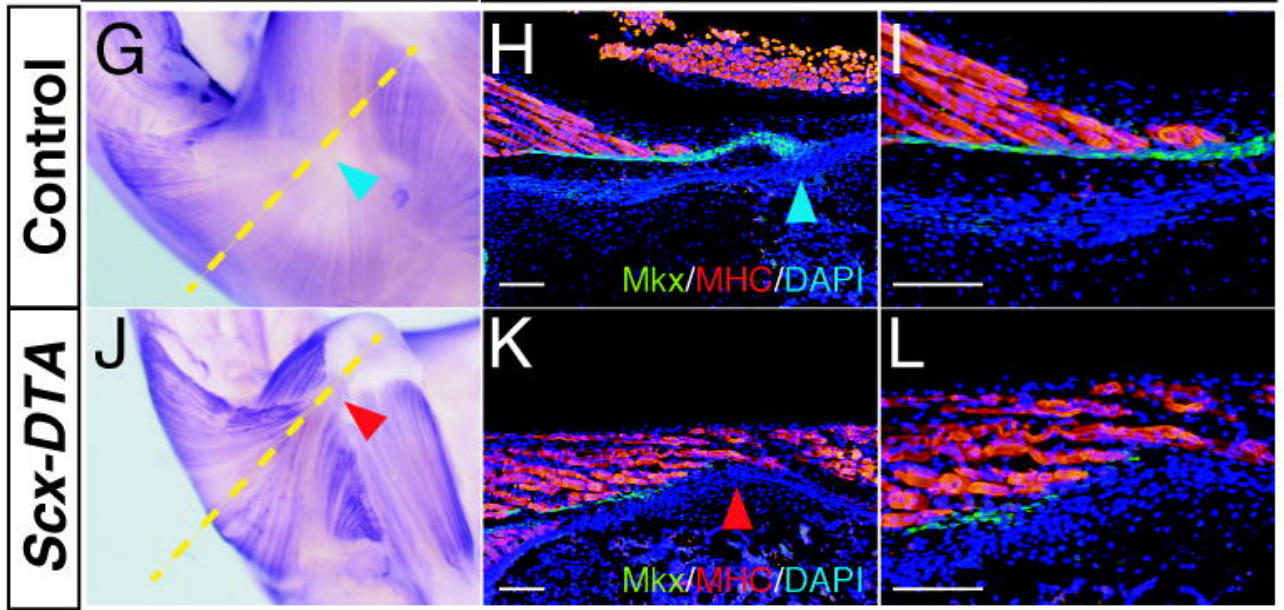
Ono et al. Supplementary Figure 1
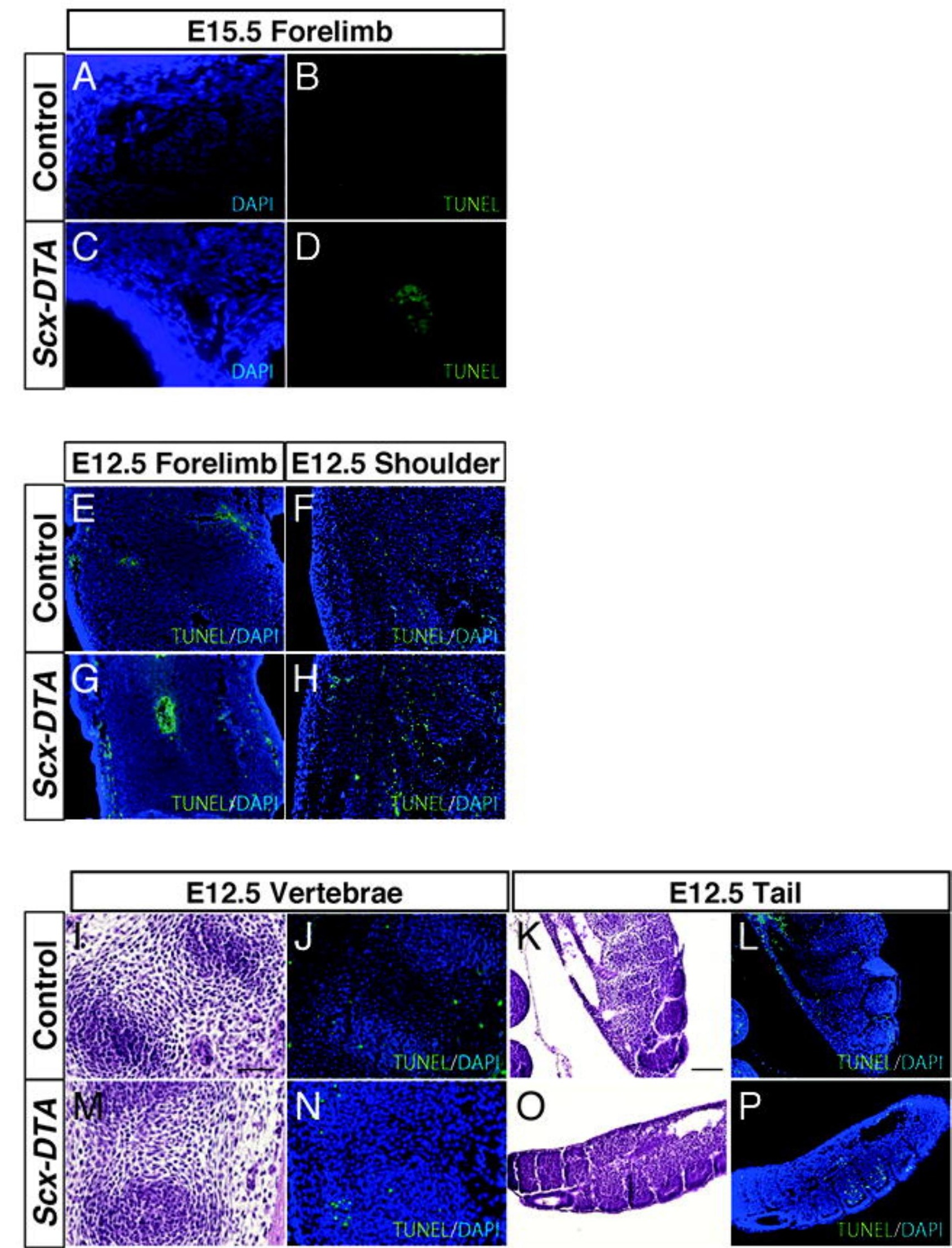


\section{Ono et al. Supplementary Figure 2}

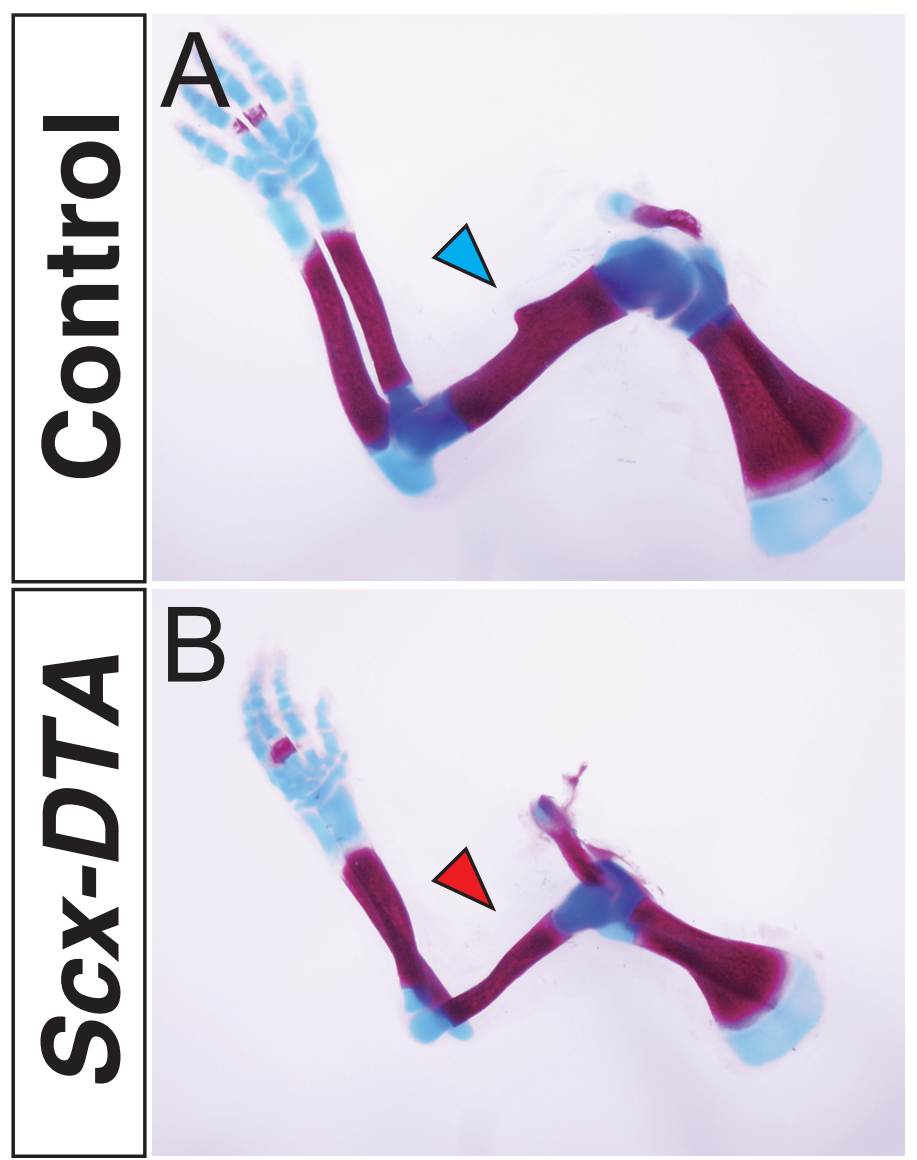


Ono et al. Supplementary Figure 3

\section{\begin{tabular}{|l|l|}
\hline P0 Whole & E15.5 Section
\end{tabular}}
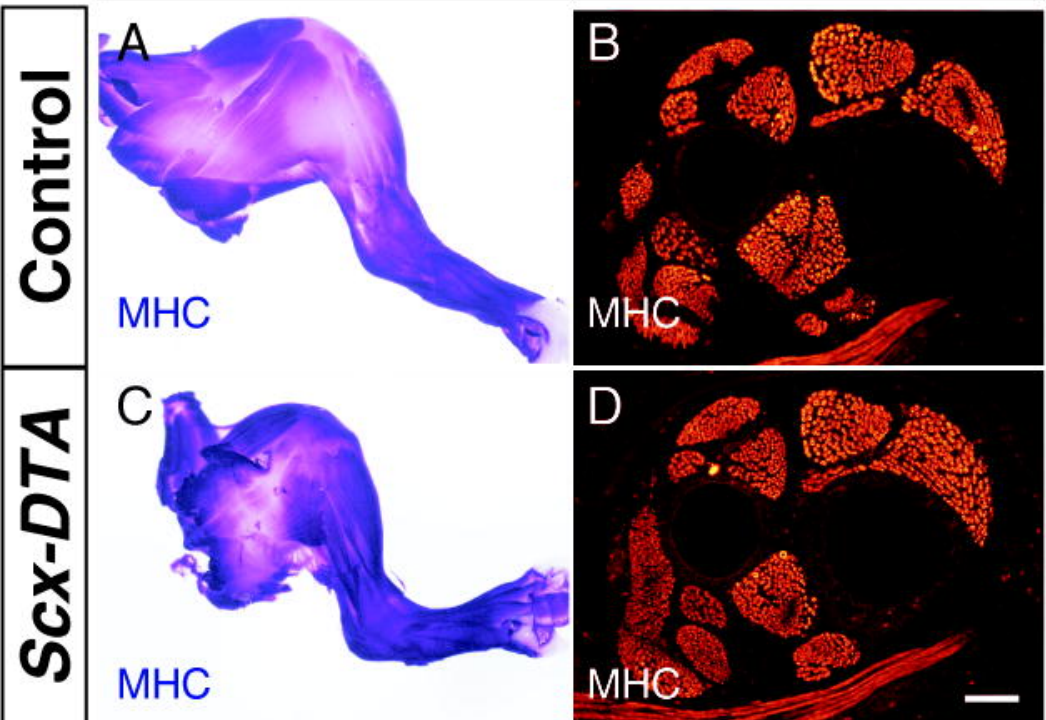

available under aCC-BY-NC-ND 4.0 International license.

\section{Ono et al. Supplementary Figure 4}

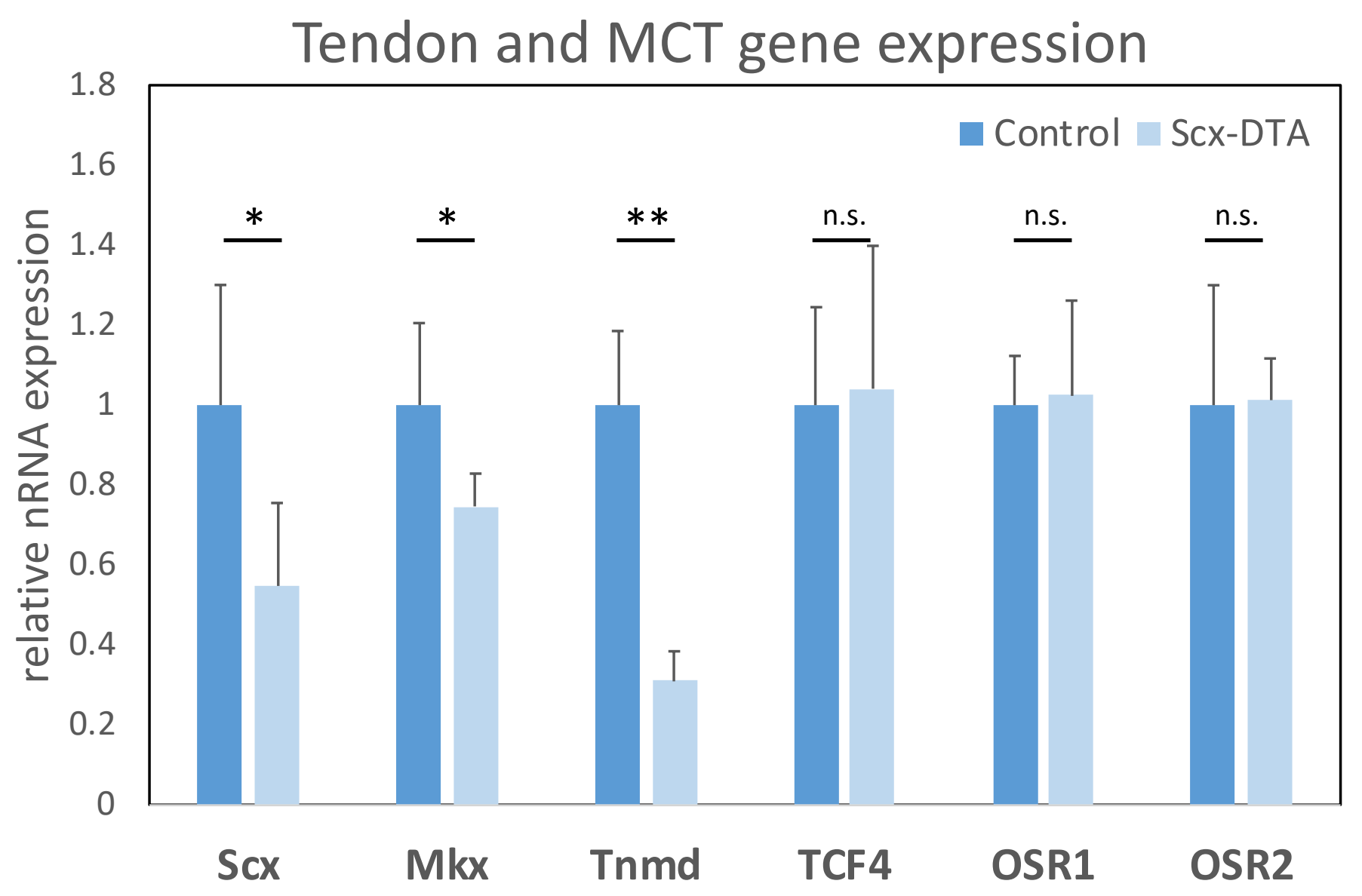


Ono et al. Supplementary Figure 5

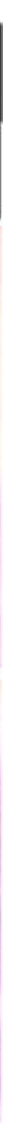


Ono et al. Supplementary Figure 6

bioRxiv preprint doi https://doi.org/10.1101/2021.01.03.443.

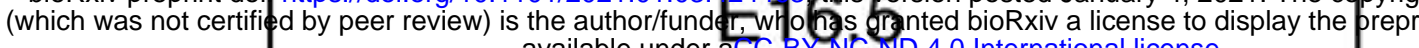
available under aCC-BY-NC-ND 4.0 international license.

\section{Pectoralis Maj. Deltoid Tuberosity}

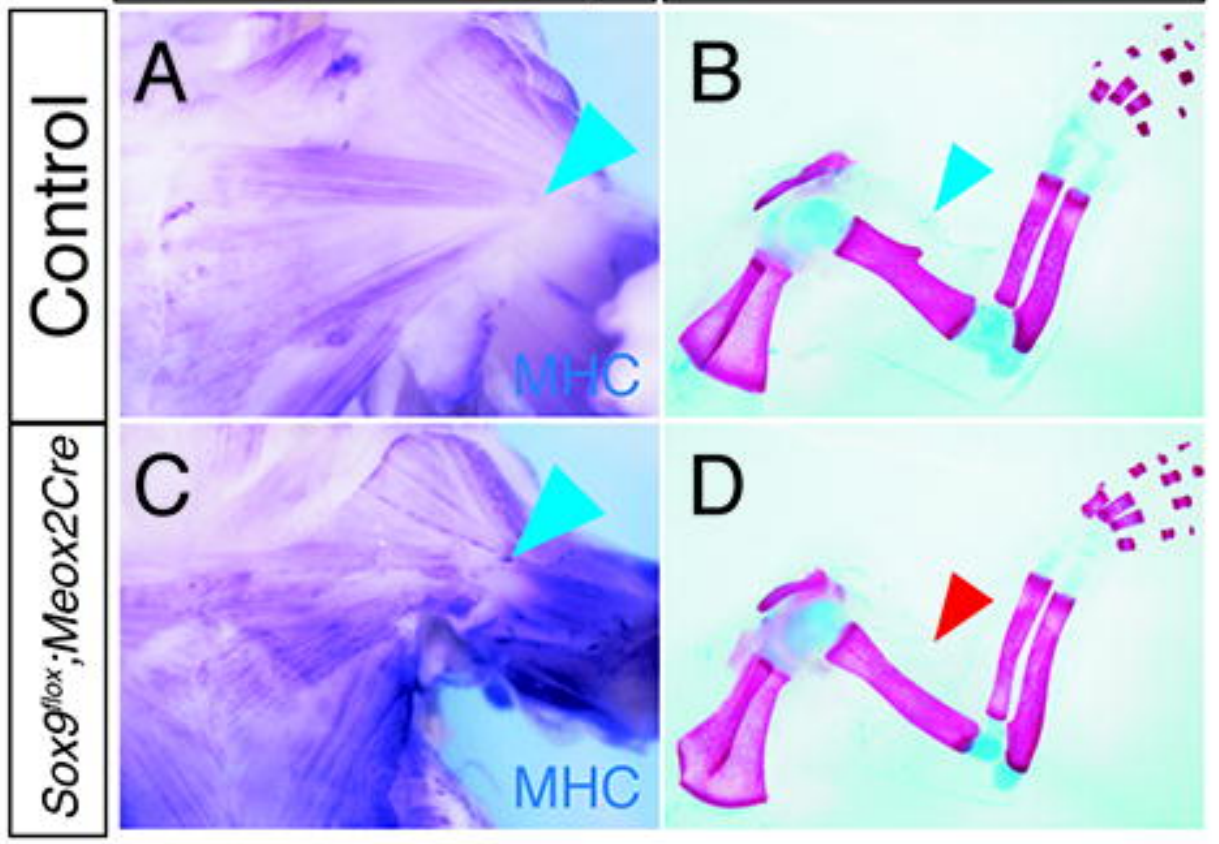

E16.5

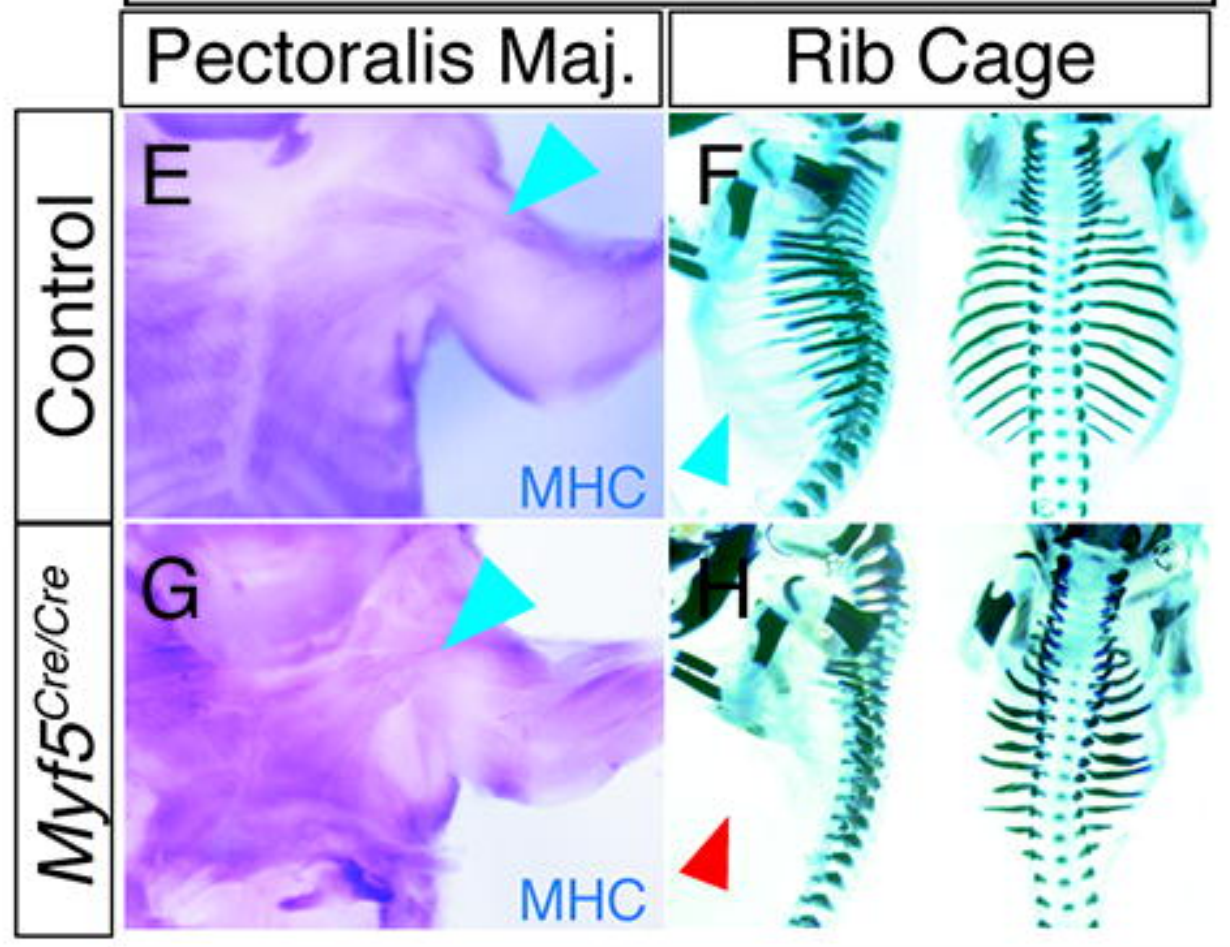

\section{E16.5}

\section{Control \\ Myf5-DTA}

\begin{tabular}{|l}
$\sum_{0}^{\circ}$ \\
.0 \\
$\bar{c}$ \\
0 \\
0 \\
0 \\
0 \\
0
\end{tabular}
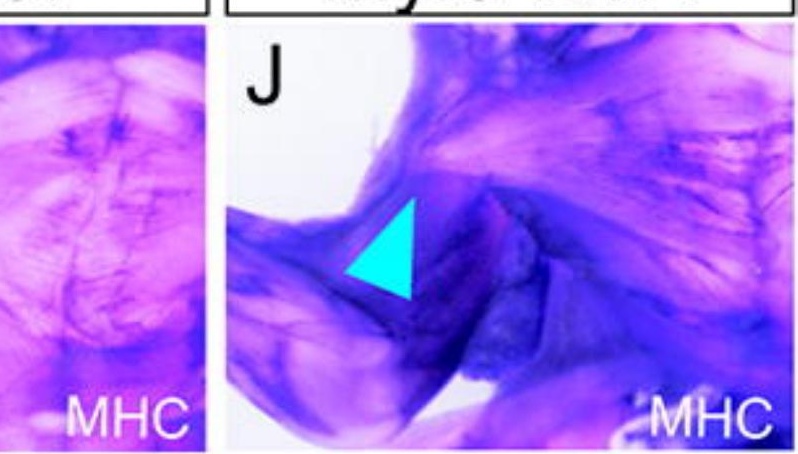
Ono et al. Supplementary Figure 7
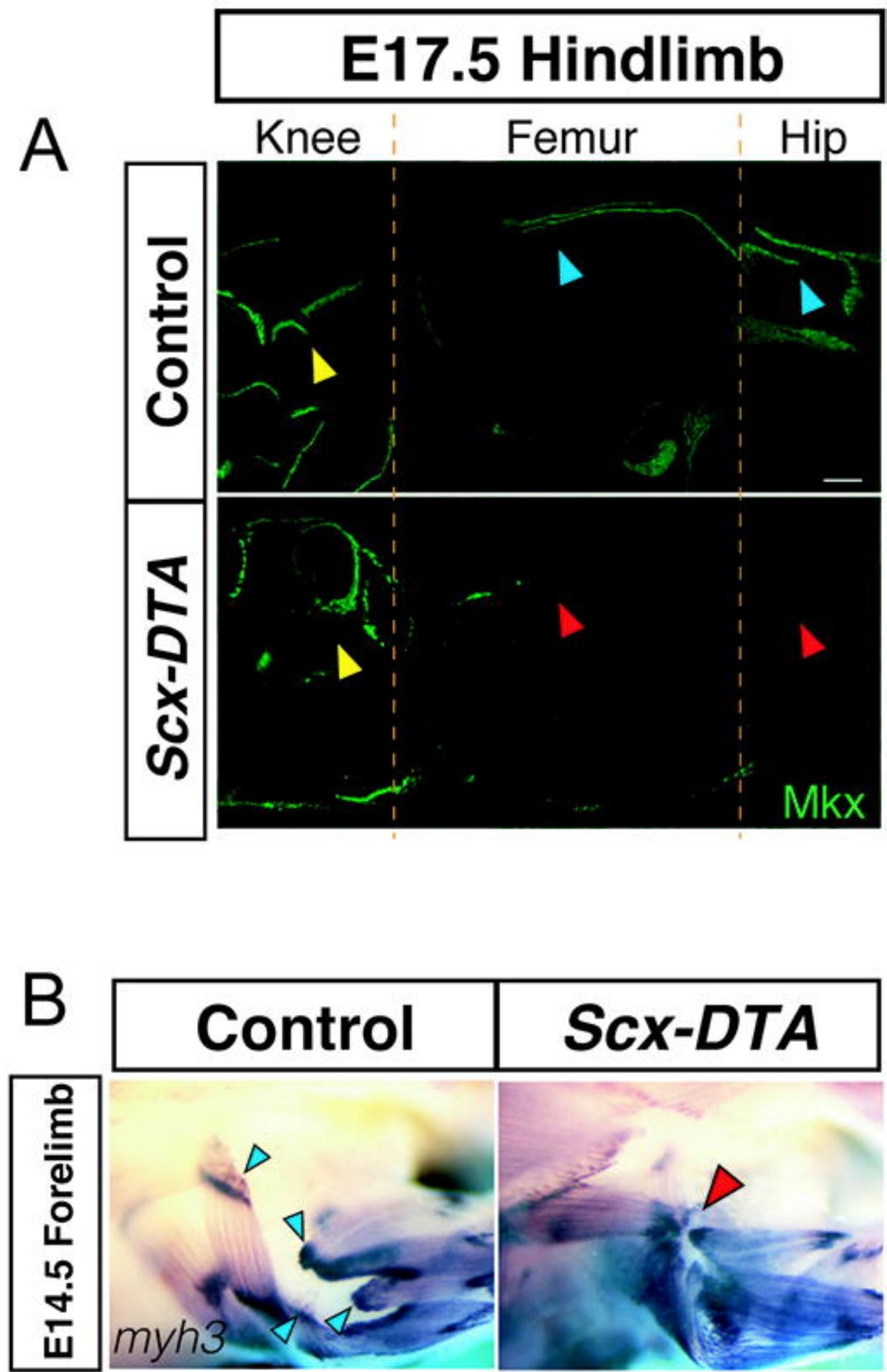